\title{
Consistent truncation on Calabi-Yau and Nearly-Kähler manifolds
}

\author{
Dimitrios Tsimpis* \\ Institut de Physique des Deux Infinis \\ Université de Lyon, UCBL, UMR 5822, CNRS/IN2P3 \\ 4 rue Enrico Fermi, 69622 Villeurbanne Cedex, France \\ E-mail: tsimpiseipnl.in2p3.fr
}

\begin{abstract}
We complete and extend the analysis of arXiv:1903.10504 in several directions: we put the 4d theory, arising from the IIA consistent truncation of the universal sector of Calabi-Yau compactification, in a form manifestly consistent with $4 \mathrm{~d} \mathscr{N}=2$ supergravity. We go beyond the universal sector and construct the $4 \mathrm{~d}$ effective action of IIA compactified on Calabi-Yau's with $h^{1,1}=1$, $h^{2,1} \geq 1$, in the presence of background flux and fermionic condensates. For ALE gravitational instantons, we show that the putative quartic gravitino condensate is non-negative, as required for the existence of (formal) de Sitter solutions of the $4 d$ theory. We discuss some of the issues in promoting these formal solutions to full-fledged string theory de Sitter solutions. We also extend the Nearly-Kähler consistent truncation of arXiv:1810.06344 to the complete bosonic sector of one vector multiplet and one hypermultiplet.
\end{abstract}

Corfu Summer Institute 2019 "School and Workshops on Elementary Particle Physics and Gravity" (CORFU2019)

31 August - 25 September 2019

Corfu, Greece

${ }^{*}$ Speaker. 


\section{Introduction}

A consistent truncation (CT) of a higher-dimensional theory $S$ is a lower-dimensional theory $S^{\prime}$ with the property that all solutions of $S^{\prime}$ also lift to solutions of $S$. CT's have been constructed on homogeneous spaces $[1,2,3,4,5,6]$, using a variety of different approaches: left-invariant forms on cosets [7, 8, 9, 10,11], $G$-structures [12, 13, 14, 15, 16, 17], double field theory and exceptional generalized geometry $[18,19,20,21,22,23,24,25,26,27,28,29]$.

In $[30,31]$ we initiated the study of CT's in the presence of fermionic condensates. As we review in the following, this approach offers a way to fix the dependence of the condensates on the moduli of the theory. This can be interesting for scenarios where such condensates play an important role, notably in potentially generating a positive cosmological constant.

In [30] we constructed a universal CT on Nearly-Kähler (NK) manifolds in the presence of dilatino condensates. "Universal" means that the CT is valid for any NK manifold, independently of whether it is homogeneous, or whether it admits a coset description. ${ }^{1}$ CT's on homogeneous NK manifolds have been constructed before (in the absence of condensates), relying on the coset description of these spaces [13]. Moreover, a universal reduction on NK spaces leading to fourdimensional $\mathscr{N}=2$ gauged supergravity, has been performed in [35], but without a proof of consistency. $^{2}$ In the case where the NK manifold is topologically an $S^{6}$, the consistency of [35] was shown in [45].

In [31] we constructed a universal CT of IIA compactified on Calabi-Yau (CY) manifolds, in the presence of background flux and gravitino condensates, comprising the gravity multiplet, one vectormultiplet, and one hypermultiplet. The effective $4 \mathrm{~d}$ action resulting from type II compactification on CY manifolds has been constructed before both in the absence [46, 47] and in the presence of background flux [48], and the consistency of the resulting 4d theory with (gauged) $\mathscr{N}=2$ supergravity has been established $[49,50,51,52]$. Beyond the universal sector, these $4 \mathrm{~d}$ effective actions may not be CT's in general.

In the present paper we complete and extend the analysis of [30, 31] in several directions. In section 2 we put the $4 \mathrm{~d}$ theory, arising from the IIA consistent truncation of the universal sector of Calabi-Yau compactification, in a form manifestly consistent with $4 \mathrm{~d} \mathscr{N}=2$ supergravity. In section 3 we go beyond the universal sector and construct the 4 d effective action of IIA compactified on Calabi-Yau's with $h^{1,1}=1, h^{2,1} \geq 1$, in the presence of background flux and fermionic condensates. The $4 \mathrm{~d}$ actions thus derived are valid for generic gravitino condensates arising from gravitational instanton backgrounds with positive-chirality $4 \mathrm{~d}$ zero modes. Specializing to the case of ALE gravitational instantons, in section 4 we show that the putative quartic gravitino condensate is non-negative, as required for the existence of (formal) de Sitter solutions of the $4 \mathrm{~d}$ theory [31].

\footnotetext{
${ }^{1}$ There exist four homogeneous compact NK six-manifolds: $S^{6}, S^{3} \times S^{3}, \mathbb{C P}^{3}$ and $\mathbb{F}^{1,2}$ [32], and these were until recently the only known compact NK six-manifolds. In [33] two non-homogenous examples were constructed, and it is has been argued that many more should exist [34].

${ }^{2}$ The author of [35] follows the approach of [36, 37, 38, 39, 40, 41], whereby one postulates the existence of a certain finite set of forms on the internal manifold, and constructs a reduction ansatz based on these forms. Inserting the ansatz in the higher-dimensional action and integrating over the internal directions can then be shown to result in a lowerdimensional gauged supergravity. In general, this procedure is not guaranteed to lead to a CT [41, 42]. In addition to showing the compatibility of the reduction with gauged supergravity, [35] proves that certain supersymmetric solutions of the $4 \mathrm{~d}$ theory uplift to a well-known class of solutions $[43,44]$ of the $10 \mathrm{~d}$ theory.
} 
We discuss some of the issues in promoting these formal solutions to full-fledged string theory de Sitter solutions in section 6. In section 5 we also extend the Nearly-Kähler consistent truncation of [30] to the complete bosonic sector of one vector multiplet and one hypermultiplet.

\section{CY universal truncation}

Let us recall the consistent truncation of [31]: it is obtained from massless IIA supergravity by the following form ansatz, cf. appendices A, B for our conventions,

$$
\begin{aligned}
& F=\mathrm{d} \alpha ; \quad H=\mathrm{d} \chi_{\wedge} J+\mathrm{d} \beta+\frac{1}{2} \operatorname{Re}\left(b_{0} \Omega^{*}\right) \\
& G=\varphi \mathrm{vol}_{4}+\frac{1}{2} c_{0} J_{\wedge} J+J_{\wedge}(\mathrm{d} \gamma-\alpha \wedge \mathrm{d} \chi)-\frac{1}{2} D \xi_{\wedge} \operatorname{Im} \Omega-\frac{1}{2} D \xi_{\wedge}^{\prime} \operatorname{Re} \Omega,
\end{aligned}
$$

where $\alpha, \gamma$ are $4 \mathrm{~d}$ one-forms; $\beta$ is a $4 \mathrm{~d}$ two-form dual to a scalar $b$ (see (2.7) below for the precise relation); $\varphi, \chi, \xi, \xi^{\prime}$ are $4 \mathrm{~d}$ scalars (of these $\varphi$ turns out to be auxiliary and will be eliminated by its equations of motion); $c_{0} \in \mathbb{R}$ and $b_{0} \in \mathbb{C}$ are background fluxes; $J$ and $\Omega$ are the CY Kähler form and holomorphic three-form respectively. The covariant derivatives are defined as follows,

$$
D \xi:=\mathrm{d} \xi+b_{1} \alpha ; \quad D \xi^{\prime}:=\mathrm{d} \xi^{\prime}+b_{2} \alpha,
$$

where we set $b_{0}=i b_{1}+b_{2}$. The expressions for the forms $F, H, G$ above are such that the $10 \mathrm{~d}$ Bianchi identities (B.7) are automatically satisfied for vanishing mass. The ansatz for the metric reads,

$$
\mathrm{d} s_{(10)}^{2}=e^{2 A(x)}\left(e^{-8 A(x)} g_{\mu \nu} \mathrm{d} x^{\mu} \mathrm{d} x^{v}+g_{m n} \mathrm{~d} y^{m} \mathrm{~d} y^{n}\right),
$$

where $A$ is a scalar depending only on the $4 \mathrm{~d}$ coordinates $x^{\mu}$. The $4 \mathrm{~d}$ field content corresponds to the bosonic sector of $\mathscr{N}=2,4 \mathrm{~d}$ supergravity with a gravity multiplet $\left(g_{\mu \nu}, \alpha\right)$, one vector multiplet $(\gamma, A, \chi)$, and one hypermultiplet $\left(\phi, b, \xi, \xi^{\prime}\right) .^{3}$

The ansatz is supplemented by $4 \mathrm{~d}$ gravitino condensates arising potentially from gravitational instantons supporting spin-3/2 zero modes. We start from the following $10 \mathrm{~d}$ gravitino truncation,

$$
\Psi_{m}=0 ; \quad \Psi_{\mu+}=\psi_{\mu+} \otimes \eta ; \quad \Psi_{\mu-}=\psi_{\mu+}^{\prime} \otimes \eta^{c},
$$

where we have Wick-rotated to Euclidean signature; $\eta$ is the (normalized) covariantly-constant spinor associated with the CY metric $g_{m n}$; the $4 \mathrm{~d}$ gravitini $\psi_{\mu+}, \psi_{\mu+}^{\prime}$ are assumed to have positive chirality, as e.g. in the case of ALE gravitational instantons. They are not dynamical: they should be thought of as zero modes of the Dirac operator in the background of the gravitational instanton. The condensates are then expressed in terms of two "parameters" $\mathscr{A}, \mathscr{B}$ corresponding to the quadratic and quartic condensates respectively, ${ }^{4}$

$$
\begin{aligned}
\mathscr{A} & :=\left(\tilde{\psi}_{\mu+} \gamma^{\mu v} \psi_{v+}^{\prime}\right)=-\left(\tilde{\psi}_{+}^{\mu} \psi_{\mu+}^{\prime}\right) \\
\mathscr{B} & :=-\frac{3}{2}\left(\tilde{\psi}_{[\mu} \psi_{v]}^{\prime}\right)^{2}+\left(\tilde{\psi}^{\mu} \gamma^{\rho v} \psi_{\mu}^{\prime}\right)\left(\tilde{\psi}_{\rho} \psi_{v}^{\prime}\right)+3\left(\tilde{\psi}_{\left[\mu_{1}\right.} \gamma_{\mu_{2} \mu_{3}} \psi_{\left.\mu_{4}\right]}^{\prime}\right)^{2} .
\end{aligned}
$$

\footnotetext{
${ }^{3}$ More precisely, the $\mathscr{N}=2$ multiplets should be expressed in terms of volume modulus $v$, the $4 \mathrm{~d}$ dilaton $\hat{\phi}$ and the vector-multiplet vector $\tilde{\gamma}$, cf. section 2.1 .

${ }^{4}$ Note however that $\mathscr{A}, \mathscr{B}$ have a $4 \mathrm{~d}$ metric dependence, due to the contractions between vector indices of the gravitini.
} 
We emphasize that these expressions are valid for all gravitational instantons with positive-chirality gravitino zero modes: they are not limited to ALE gravitational instantons.

As was shown in [31], substituting the truncation ansatz (2.1)-(2.5) into the $10 \mathrm{~d}$ equations of motion of appendix B, results in a set of $4 \mathrm{~d}$ equations, all of which are derivable from a single $4 \mathrm{~d}$ action, thereby proving the consistency of the truncation. The $4 \mathrm{~d}$ action is given by,

$$
\begin{aligned}
S_{4}=\int \mathrm{d}^{4} x \sqrt{g}\left(R-24(\partial A)^{2}-\frac{1}{2}(\partial \phi)^{2}-\frac{3}{2} e^{-4 A-\phi}(\partial \chi)^{2}-\frac{1}{2} e^{-6 A+\phi / 2}\left[(D \xi)^{2}+\left(D \xi^{\prime}\right)^{2}\right]\right. \\
\left.-\frac{1}{2} e^{\phi-12 A}(D b)^{2}-\frac{1}{4} e^{3 \phi / 2+6 A} \mathrm{~d} \alpha^{2}-\frac{3}{4} e^{\phi / 2+2 A}(\mathrm{~d} \gamma-\alpha \wedge \mathrm{d} \chi)^{2}-V\right)+\int 3 \chi \mathrm{d} \gamma_{\wedge} \mathrm{d} \gamma
\end{aligned}
$$

where we have dualized the two-form $\beta$ to a scalar $b$, the "axion", via the relation,

$$
\mathrm{d} \beta=e^{\phi-12 A} \star_{4} D b ; \quad D b:=\left[\mathrm{d} b+\frac{1}{2}\left(\xi \mathrm{d} \xi^{\prime}-\xi^{\prime} \mathrm{d} \xi\right)+3 c_{0}(\gamma-\chi \alpha)+\Xi \alpha\right],
$$

with $\Xi:=b_{2} \xi-b_{1} \xi^{\prime}$. The potential of the theory is given by,

$$
\begin{aligned}
V\left(\chi, \xi, \xi^{\prime}, \phi, A\right) & =\frac{3}{2} c_{0}^{2} e^{\phi / 2-14 A}+\frac{1}{2}\left|b_{0}\right|^{2} e^{-\phi-12 A} \\
& -3 c_{0} \mathscr{A} e^{\phi / 4-4 A}+e^{6 A} \mathscr{B}+\frac{1}{2}\left(\mathscr{A} e^{3 A}+\left(3 c_{0} \chi-\Xi\right) e^{-\phi / 4-9 A}\right)^{2}
\end{aligned}
$$

Note that for $c_{0} \neq 0,(2.6)$-(2.8) are invariant under the shift,

$$
\chi \rightarrow \chi+\frac{e_{0}}{3 c_{0}} ; \quad \Xi \rightarrow \Xi+e_{0},
$$

where $e_{0}$ is an arbitrary real constant. Interestingly, setting the background flux $b_{0}, c_{0}$ to zero after performing the shift (2.9), still results in a gauged theory provided $e_{0} \neq 0$ [48].

\section{Euclidean signature}

Instantons are solutions of the action in Euclidean signature. However, as is well-known, the operation of dualization does not commute with Wick rotation [53]. Starting from the Euclidean action in terms of the two-from $\beta$ and then dualizing to the axion $b$, one obtains an action which differs form (2.6) in the sign of the kinetic term for $b$.

\subsection{Comparison with $\mathscr{N}=2,4 \mathrm{~d}$ supergravity}

Let us introduce a complex scalar $t$ and the real scalars $v, \hat{\phi}$ defined as follows,

$$
t:=\chi+i v ; \quad v:=e^{2 A+\phi / 2} ; \quad \hat{\phi}:=\frac{1}{4} \phi-3 A .
$$

As we will see in the following $v$ and $\hat{\phi}$ will be identified with the volume modulus and the $4 \mathrm{~d}$ dilaton respectively. Moreover we introduce a new vector $\tilde{\gamma}$ and its field-strength,

$$
\tilde{\gamma}:=\gamma-\chi \alpha ; \tilde{\mathscr{F}}:=\mathrm{d} \tilde{\gamma},
$$

so that $\mathrm{d} \gamma-\alpha \wedge \mathrm{d} \chi=\tilde{\mathscr{F}}+\chi \mathscr{F}$, where $\mathscr{F}:=\mathrm{d} \alpha$. In terms of the new fields, the action (2.6) reads,

$$
\begin{aligned}
S_{4}=\int & \mathrm{d}^{4} x \sqrt{g_{4}}\left(R-2 \partial_{t} \partial_{t} K_{V}|\mathrm{~d} t|^{2}-2(\mathrm{~d} \hat{\phi})^{2}-\frac{1}{2} e^{4 \hat{\phi}}(D b)^{2}-\frac{1}{2} e^{2 \hat{\phi}}\left|D \xi^{\prime}+i D \xi\right|^{2}\right. \\
& \left.-\frac{1}{4} v^{3} \mathscr{F}^{2}-\frac{3}{4} v(\tilde{\mathscr{F}}+\chi \mathscr{F})^{2}-V\right)+\int 3 \chi \tilde{\mathscr{F}} \wedge \tilde{\mathscr{F}}+\chi^{3} \mathscr{F} \wedge \mathscr{F}+3 \chi^{2} \mathscr{F} \wedge \tilde{F}
\end{aligned}
$$


where we have introduced the vector-multiplet Kähler potential,

$$
K_{V}=-3 \ln (t-\bar{t})+\text { const . }
$$

It can be verified that the action (2.12) is of the form of $\mathscr{N}=2$ supergravity, cf. (C.11). To see this, first note that there is only one complexified Kähler modulus, so that $I=0,1$ in the formulae of Appendix C. Accordingly we may drop the $i$-index in (C.1), so that the $t^{i}$ variable therein is identified with the $t$ of (2.13). We may choose the unique harmonic (1,1)-form on the CY so that the triple intersection is normalized to $\mathscr{K}_{i j k}=6$, cf. (C.2). Taking (2.10) into account, we then find $\mathscr{K}=v^{3}$, so that (C.3) precisely reduces to (2.13), and the vector-multiplet scalar kinetic terms of (C.11) reduce to those of (2.12). Moreover, taking (2.10) into account, eq. (C.4) gives,

$$
\mathfrak{R} \mathscr{N}=\left(\begin{array}{cc}
2 \chi^{3} & 3 \chi^{2} \\
3 \chi^{2} & 6 \chi
\end{array}\right) ; \mathfrak{I} \mathscr{N}=\left(\begin{array}{cc}
v^{3}+3 v \chi^{2} & 3 v \chi \\
3 v \chi & 3 v
\end{array}\right) .
$$

Substituting in eq. (C.11) then reproduces precisely the gauge kinetic and Chern-Simons terms in (2.12), provided we identify $\alpha, \tilde{\gamma}$ with $A^{I=0}, A^{I=1}$ respectively. The corresponding Killing vectors can then be read off by comparing (2.2), (2.7) with (C.13),

$$
k_{I=0}^{u} \partial_{q^{u}}=-\Xi \partial_{b}-b_{1} \partial_{\xi}-b_{2} \partial_{\xi^{\prime}} ; \quad k_{I=1}^{u} \partial_{q^{u}}=-3 c_{0} \partial_{b},
$$

where the $q^{u}$ coordinates are identified with $\hat{\phi}, b, \xi, \xi^{\prime}$, and we took into account that there is only one hypermultiplet: $n_{H}=1$, so that $u=1, \ldots, 4$. To compare with the quaternionic metric (C.12) first note that there are no complex structure deformations, so that the $z^{a}$ 's are absent in our case, and the hypermultiplet indices take on a single value, $A, B=0$. The periods are then calculated from $\mathscr{G}=-\frac{i}{2}\left(Z^{0}\right)^{2}$, evaluated at $Z^{0}=1$, so that $\Omega=\alpha+i \beta$ in terms of the basis in (C.5). This then gives $\mathscr{M}_{A B}=-i$, and (C.12) precisely reduces to the kinetic $\hat{\phi}, b, \xi, \xi^{\prime}$ terms in (2.12). Finally, to show that the potential (2.8) is of the from given in (C.14), we compute,

$$
h_{u v} k_{I}^{u} k_{J}^{v}=\frac{1}{2}\left(\begin{array}{cr}
\left.e^{4 \hat{\phi}} \Xi^{2}+e^{2 \hat{\phi}}\left|b_{0}\right|^{2}\right) & -3 c_{0} e^{4 \hat{\phi}} \Xi \\
-3 c_{0} e^{4 \hat{\phi}} \Xi & 9 c_{0}^{2} e^{4 \hat{\phi}}
\end{array}\right),
$$

and,

$$
X^{(I} \bar{X}^{J)}=\left(\begin{array}{cc}
1 & \chi \\
\chi & \chi^{2}+v^{2}
\end{array}\right) ; \quad \mathscr{K}\left(\mathfrak{I} \mathscr{N}^{-1}\right)^{I J}=-\left(\begin{array}{cc}
1 & \chi \\
\chi & \chi^{2}+\frac{1}{3} v^{2}
\end{array}\right) .
$$

Moreover, taking (C.15) into account, (2.15), (C.16) imply,

$$
\vec{P}_{I=1}=\left(0,0,-\frac{3}{\sqrt{2}} c_{0} e^{2 \hat{\phi}}\right) .
$$

Substituting (2.16)-(2.18) into (C.14) reproduces precisely the potential given in (2.8), in the case of vanishing condensates: $\mathscr{A}=\mathscr{B}=0$.

\section{CY compactification with $h^{1,1}=1, h^{2,1} \geq 1$}

We will now extend the universal sector truncation of section 2, to include an arbitrary number of hypermultiplets. In order to obtain the $4 \mathrm{~d}$ theory we will simply insert the new ansatz, eqs. (3.1), 
(3.2), (3.8) below, into the 10d action, keeping up to and including quadratic terms in the fluctuations. This is the standard procedure in the literature when it comes to CY compactification: it should lead to a low-energy effective action, but not necessarily to a consistent truncation [41, 42]. Nevertheless we will see that it does satisfy certain consistency requirements: (a) the resulting $4 \mathrm{~d}$ Lagrangian contains the consistent truncation of [31] as a subsector, plus terms that depend on the additional hypermultiplet scalars of the present paper; (b) the kinetic terms of the additional hypermultiplet scalars are of the standard form; (c) the resulting Lagrangian can be cast in the form of the bosonic sector of a theory with manifest $\mathscr{N}=2, d=4$ supergravity.

The ansatz for the ten-dimensional metric reads,

$$
\mathrm{d} s_{(10)}^{2}=e^{2 A(x)}\left(e^{-8 A(x)} g_{\mu \nu}(x) \mathrm{d} x^{\mu} \mathrm{d} x^{\nu}+g_{m n}(x, y) \mathrm{d} y^{m} \mathrm{~d} y^{n}\right),
$$

where the scalar $A$ only depends on the four-dimensional coordinates $x^{\mu}$, but not on the $y^{m}$ coordinates of the CY. Contrary to the consistent truncation ansatz of [31], the internal CY metric $g_{m n}$ is now allowed to vary as we move along the four-dimensional spacetime. Explicitly we set,

$$
g_{m n}(x, y):=\stackrel{\circ}{g}_{m n}(y)+\delta g_{m n}(x, y) ; \quad \delta g_{m n}(x, y):=\frac{6}{|\grave{\Omega}|^{2}}\left(\delta z^{a}(x)\left(\Phi_{a}\right)_{m}^{p q} \stackrel{\circ}{\Omega}_{n p q}^{*}+\text { c.c. }\right),
$$

where $a=1, \ldots, h^{2,1}$. The metric is thus expressed as a fluctuation around a fiducial CY metric $\stackrel{\circ}{g}_{m n}(y)$. We may think of the latter as being defined at a point $\dot{z}^{a}$ in the (complex) $h^{2,1}$-dimensional space of complex structure moduli $\mathfrak{M}_{c}$, while the nearby metric $g_{m n}$ is defined at the point $\stackrel{\circ}{a}^{a}+\delta z^{a}$. The variations $\delta z^{a}$ span the cotangent space of $\mathfrak{M}_{c}$ at the point $\stackrel{i}{a}^{a}$, and the fluctuation $\delta g_{m n}(x, y)$ is a complex-structure deformation. The $\Phi_{a}$ 's, $a=1, \ldots, h^{2,1}$, consitute a basis of harmonic $(2,1)$ forms, with respect to the metric and complex structure at the point $z^{a}{ }^{5}$ Furthermore we will assume that the volume of the metric $g_{m n}$ is constant, so that the volume modulus of the compactification space is given by the scalar $v$ of (2.10), as in the previous section. The complete moduli space $\mathfrak{M}$ of the compactification has a direct-product structure:

$$
\mathfrak{M}=\mathfrak{M}_{k} \times \mathfrak{M}_{c},
$$

where $\mathfrak{M}_{k}$ is the (real) one-dimensional moduli space of Kähler deformations parametrized by the volume modulus.

We denote by $(J, \Omega ̊)$ the Kähler and holomorphic forms of the fiducial CY, defined at the point $\stackrel{\circ}{z}^{a}$, while those of the nearby CY, defined at the point $\stackrel{i}{z}^{a}+\delta z^{a}$, will be denoted by $(J, \Omega){ }^{6}$ Since the

${ }^{5}$ The metric deformation can be viewed as resulting from a variation of the holomorphic top form,

$$
\delta \Omega=\delta z^{a} \Phi_{a},
$$

with the induced deformation of complex structure given by,

$$
\delta \mathscr{I}_{m}{ }^{n}=\frac{6 i}{|\Omega|^{2}}\left(\delta z^{a}\left(\Phi_{a}\right)_{m p q} \bar{\Omega}^{n p q}-\delta \bar{z}^{a}\left(\bar{\Phi}^{a}\right)^{n p q} \Omega_{m p q}\right) .
$$

The associated metric variation, $\delta g_{m n}=-\delta \mathscr{I}_{m}{ }^{q} J_{q n}$, is then given by (3.2). Note that the right-hand side of that equation is automatically symmetric in $(m, n)$.

${ }^{6}$ In the present paper, to conform with the standard treatment of complex structure deformations, we do not impose a normalization on $\Omega$. Compared to the conventions of [31]: $\Omega^{\text {there }}=\frac{4 \sqrt{3}}{\left|\Omega^{\text {here }}\right|} \Omega^{\text {here }}$. 
CY metric $g_{m n}$ is assumed to have fixed volume, and its associated Kähler form does not change under complex structure deformations, we have $J=J$. It can be seen that $\delta g_{m n}$ given in (3.2) is a solution to the six-dimensional Ricci-flatness condition $R_{m n}(\stackrel{\circ}{g}+\delta g)=0$, to linear order in $\delta z^{a}$.

Plugging the metric ansatz into the $10 \mathrm{~d}$ Einstein term we obtain,

$$
\begin{aligned}
\sqrt{g_{10}} R_{10}=\sqrt{g_{6}} \sqrt{g_{4}}\left(R_{4}-24(\partial A)^{2}-g^{\mu \nu} \hat{\nabla}_{\mu}\left(g^{m n} \partial_{\nu} \delta g_{m n}\right)\right. \\
\left.-\frac{1}{4} g^{\mu v}\left[g^{m n} g^{p q} \partial_{\mu} \delta g_{m p} \partial_{\nu} \delta g_{n q}+g^{m n} g^{p q} \partial_{\mu} \delta g_{m n} \partial_{\nu} \delta g_{p q}\right]\right),
\end{aligned}
$$

where we have taken into account the Ricci-flatness of the six-dimensional metric $g_{m n}(x, y) ; \hat{\nabla}$ is the connection associated to the $4 \mathrm{~d}$ metric $\hat{g}_{\mu \nu}:=e^{-8 A(x)} g_{\mu \nu}$. So far equation (3.4) is exact, in that the deformations $\delta g$ do not need to be infinitesimal. Moreover the internal metric depends on the $4 \mathrm{~d}$ spacetime only through the fluctuation, $\partial_{v} \delta g_{m n}=\partial_{v} g_{m n}$, so that $g^{m n} \partial_{v} \delta g_{m n}=\partial_{v} \ln g_{6}=0$, where in the last equality we took into account that $g_{m n}$ has fixed volume, cf. the discussion below (3.2). Since eq. (3.4) is already quadratic in the fluctuations, we may use the linearized expressions (3.2) for $\delta g_{m n}$. To quadratic order in $\delta z^{a}$, eq. (3.4) then simplifies to,

$$
\int \sqrt{g_{10}} R_{10}=V_{6} \int \sqrt{g_{4}}\left(R_{4}-24(\partial A)^{2}-2 g_{a \bar{b}} \mathrm{~d} z^{a} \mathrm{~d} \bar{z}^{b}\right)
$$

where we have defined,

$$
g_{a \bar{b}}:=-\frac{\int \Phi_{a^{\wedge}} \bar{\Phi}_{b}}{\int \Omega_{\wedge} \Omega^{*}}
$$

and we have used that $\partial_{\mu} \delta z^{a}=\partial_{\mu} z^{a}$. Moreover we have taken (A.7) into account, and introduced the $\mathrm{CY}$ volume $V_{6}:=\int \operatorname{vol}_{6}$ which is constant, cf. the discussion below (3.2).

Note that as long as we are only keeping quadratic terms in the fluctuations, it does not matter whether we use $\Omega$ or $\Omega$ in (3.6). However, using $\Omega$ leads to a covariant expression with respect to the moduli. Indeed, in accordance with the standard form for the kinetic terms of complex-structure moduli, $g_{a \bar{b}}$ can be viewed as a metric derived from a Kähler potential, ${ }^{7}$

$$
g_{a \bar{b}}=\partial_{z^{a}} \bar{\partial}_{z^{b}} K_{H} ; \quad K_{H}(z, \bar{z})=-\ln \left(i \int \Omega \wedge \Omega^{*}\right) .
$$

Let us now consider the ansatz for the forms. These are expanded so that the $10 \mathrm{~d}$ Bianchi identities, eq. (B.7) with zero mass, are automatically satisfied,

$$
\begin{aligned}
& F=\mathrm{d} \alpha ; \quad H=\mathrm{d} \chi_{\wedge} J+\mathrm{d} \beta+p^{A} \alpha_{A}+q_{A} \beta^{A} \\
& G=\varphi \operatorname{vol}_{4}+\frac{1}{2} c_{0} J_{\wedge} J+J_{\wedge}(\mathrm{d} \gamma-\alpha \wedge \mathrm{d} \chi)-\left(D \xi_{A^{\prime}}^{\prime} \beta^{A}+D \xi^{A} \alpha_{A}\right),
\end{aligned}
$$

${ }^{7}$ Recall that as we move in $\mathfrak{M}_{c}, \Omega$ varies holomorphically with respect to $z^{a}$, so that $\partial_{z^{a}} \bar{\Omega}=0$. Moreover rescalings of the form $\Omega \rightarrow f(z) \Omega$, where $f$ is an arbitrary holomorphic function of $z^{a}$, do not change the complex structure of the CY. Thus $\Omega$ may be viewed as a section of a holomorphic line bundle over $\mathfrak{M}_{c}$ [54]. Motion in $\mathfrak{M}_{c}$ is described in terms of the Kähler-covariant derivative:

$$
\mathscr{D}_{a} \Omega=\Phi_{a} ; \quad \delta \Omega=\delta z^{a} \mathscr{D}_{a} \Omega,
$$

where $\mathscr{D}_{a}:=\partial_{z^{a}}+\partial_{z^{a}} K_{H}$. This is consistent with the definition of the Kähler potential in (3.7) as can be seen by wedging both sides of the first equation above with $\bar{\Omega}$ and integrating over the CY. The consistency with (3.6) follows similarly. 
where we have expanded on the basis (C.5), and introduced background charges $p^{A}, q_{A} \in \mathbb{R}$, following the notation of [48]; the index $A$ is related to the index $a$ in (3.3) via $A=(0, a)$, so that $A=0,1, \ldots, h^{2,1}$. The covariant derivatives are given by,

$$
D \xi^{A}:=\mathrm{d} \xi^{A}+p^{A} \alpha ; \quad D \xi_{A}^{\prime}:=\mathrm{d} \xi_{A}^{\prime}+q_{A} \alpha
$$

The following expressions are useful,

$$
\begin{aligned}
\star_{10} F= & \frac{1}{6} e^{6 A} \star_{4} \mathrm{~d} \alpha_{\wedge} J^{3} \\
\star_{10} H= & \left.\frac{1}{2} e^{4 A+2 B} \star_{4} \mathrm{~d} \chi_{\wedge} J^{2}+\frac{1}{6} e^{4 A-2 B} \star_{4} h_{\wedge} J^{3}+e^{-12 A}\left(p^{A} \star_{6} \alpha_{A}+q_{A} \star_{6} \beta^{A}\right)\right)_{\wedge} \operatorname{vol}_{4} \\
\star_{10} G= & -\frac{1}{6} \varphi e^{2 A-4 B} J^{3}+c_{0} e^{2 A+4 B} \operatorname{vol}_{4} J+\frac{1}{2} e^{2 A} \star_{4}(\mathrm{~d} \gamma-\alpha \wedge \mathrm{d} \chi) J^{2} \\
& -\frac{1}{2} e^{2 A+2 B} \star_{4} D \xi_{A^{\wedge} \star_{6}}^{\prime} \beta^{A}-\frac{1}{2} e^{2 A+2 B} \star_{4} D \xi^{A}{ }_{\wedge} \star_{6} \alpha_{A},
\end{aligned}
$$

where the four-dimensional Hodge-star is with respect to the unwarped metric. Moreover, taking (C.9) into account, we compute,

$$
\begin{aligned}
F^{2}= & e^{-4 A-4 B} \mathrm{~d} \alpha^{2} \\
H^{2}= & 18 e^{-6 A-2 B}(\partial \chi)^{2}+e^{-6 A-6 B} h^{2}-6 e^{-6 A}(q+\mathscr{M} \cdot p) \cdot \mathfrak{I} \mathscr{M}^{-1} \cdot\left(q+\mathscr{M}^{*} \cdot p\right) \\
G^{2}= & -24 e^{-8 A-8 B} \varphi^{2}-24 e^{-8 A-2 B}\left(D \xi^{\prime}+\mathscr{M} \cdot D \xi\right) \cdot \mathfrak{I} \mathscr{M}^{-1} \cdot\left(D \xi^{\prime}+\mathscr{M}^{*} \cdot D \xi\right) \\
& +72 c_{0}^{2} e^{-8 A}+36 e^{-8 A-4 B}(\mathrm{~d} \gamma-\alpha \wedge \mathrm{d} \chi)^{2},
\end{aligned}
$$

where the contractions on the left-hand sides above are computed with respect to the ten-dimensional metric while the contractions on the right-hand sides are taken with respect to the unwarped metric.

Plugging the above expressions into the ten-dimensional action and integrating over the internal directions, we obtain the four-dimensional Lagrangian,

$$
\begin{gathered}
S_{4}=\int \mathrm{d}^{4} x \sqrt{g}\left(R-24(\partial A)^{2}-\frac{1}{2}(\partial \phi)^{2}-2 g_{a \bar{b}} \mathrm{~d} z^{a} \mathrm{~d} \bar{z}^{b}-\frac{3}{2} e^{-4 A-\phi}(\partial \chi)^{2}\right. \\
+\frac{1}{2} e^{-6 A+\phi / 2}\left(D \xi^{\prime}+\mathscr{M} \cdot D \xi\right) \cdot \mathfrak{I} \mathscr{M}^{-1} \cdot\left(D \xi^{\prime}+\mathscr{M}^{*} \cdot D \xi\right) \\
\left.-\frac{1}{4} e^{3 \phi / 2+6 A} \mathrm{~d} \alpha^{2}-\frac{3}{4} e^{\phi / 2+2 A}(\mathrm{~d} \gamma-\alpha \wedge \mathrm{d} \chi)^{2}-\frac{1}{12} e^{-\phi+12 A} \mathrm{~d} \beta^{2}-V\right) \\
+\int 3 c_{0} \mathrm{~d}(\gamma-\alpha \chi)_{\wedge} \beta+3 \chi \mathrm{d} \gamma_{\wedge} \mathrm{d} \gamma+\Xi \beta \wedge \mathrm{d} \alpha-\beta \wedge D \xi_{A^{\wedge}}^{\prime} D \xi^{A}
\end{gathered}
$$

where the potential of the theory is given by,

$$
\begin{aligned}
V(\chi, \phi, A) & =\left(\Xi-3 c_{0} \chi\right) \varphi-e^{12 A+\phi / 4} \varphi \mathscr{A}-\frac{1}{2} e^{18 A+\phi / 2} \varphi^{2} \\
& +\frac{3}{2} c_{0}^{2} e^{\phi / 2-14 A}-\frac{1}{2}(q+\mathscr{M} \cdot p) \cdot \mathfrak{I} \mathscr{M}^{-1} \cdot\left(q+\mathscr{M}^{*} \cdot p\right) e^{-\phi-12 A} \\
& -3 c_{0} \mathscr{A} e^{\phi / 4-4 A}+e^{6 A \mathscr{B}},
\end{aligned}
$$

with $\Xi:=p^{A} \xi_{A}^{\prime}-q_{A} \xi^{A}$; the gravitino condensates were given in (2.5).

The non-dynamical scalar $\varphi$ is constrained by the ten-dimensional $G$-field equation of motion, cf. (B.6), which implies in particular,

$$
0=\mathrm{d}\left(e^{\phi / 2+18 A} \varphi+e^{\phi / 4+12 A} \mathscr{A}+3 c_{0} \chi-\Xi\right)
$$


This is consistent with the equations of motion for $\varphi$ coming from (3.12), (3.13),

$$
\varphi=e^{-\phi / 2-18 A}\left(\Xi-3 c_{0} \chi-e^{\phi / 4+12 A} \mathscr{A}\right) .
$$

Plugging the above back into (3.13), we obtain the following expression for the potential,

$$
\begin{aligned}
V\left(\chi, \xi, \xi^{\prime}, \phi, A\right) & =\frac{3}{2} c_{0}^{2} e^{\phi / 2-14 A}-\frac{1}{2}(q+\mathscr{M} \cdot p) \cdot \mathfrak{I} \mathscr{M}^{-1} \cdot\left(q+\mathscr{M}^{*} \cdot p\right) e^{-\phi-12 A} \\
& -3 c_{0} \mathscr{A} e^{\phi / 4-4 A}+e^{6 A} \mathscr{B}+\frac{1}{2}\left(\mathscr{A} e^{3 A}+\left(3 c_{0} \chi-\Xi\right) e^{-\phi / 4-9 A}\right)^{2}
\end{aligned}
$$

The ten-dimensional $H$-field equation of motion, cf. (B.6), implies,

$$
\mathrm{d}\left(e^{-\phi+12 A} \star_{4} \mathrm{~d} \beta\right)=3 c_{0}(\mathrm{~d} \gamma-\alpha \wedge \mathrm{d} \chi)-D \xi_{A}^{\prime} \wedge D \xi^{A}+e^{\phi / 2+18 A} \varphi \mathrm{d} \alpha+e^{\phi / 4+12 A} \mathscr{A} \mathrm{d} \alpha .
$$

This is consistent with the equation of motion for $\beta$ coming from (3.12), as can be seen by eliminating $\varphi$ using (3.15). Eq. (3.17) can thus be solved to trade the two-form $\beta$ for the axion $b$,

$$
\mathrm{d} \beta=e^{\phi-12 A} \star_{4}\left[\mathrm{~d} b+\frac{1}{2}\left(\xi_{A}^{\prime} \mathrm{d} \xi^{A}-\xi^{A} \mathrm{~d} \xi_{A}^{\prime}\right)+3 c_{0}(\gamma-\chi \alpha)+\Xi \alpha\right],
$$

where we have taken (3.9) into account. Using (3.18), we obtain the action in terms of the axion,

$$
\begin{aligned}
S_{4}=\int & \mathrm{d}^{4} x \sqrt{g}\left(R-24(\partial A)^{2}-\frac{1}{2}(\partial \phi)^{2}-2 g_{a \bar{b}} \mathrm{~d} z^{a} \mathrm{~d} \bar{z}^{b}-\frac{3}{2} e^{-4 A-\phi}(\partial \chi)^{2}\right. \\
& +\frac{1}{2} e^{-6 A+\phi / 2}\left(D \xi^{\prime}+\mathscr{M} \cdot D \xi\right) \cdot \mathfrak{I} \mathscr{M}^{-1} \cdot\left(D \xi^{\prime}+\mathscr{M}^{*} \cdot D \xi\right) \\
& \left.-\frac{1}{4} e^{3 \phi / 2+6 A} \mathrm{~d} \alpha^{2}-\frac{3}{4} e^{\phi / 2+2 A}(\mathrm{~d} \gamma-\alpha \wedge \mathrm{d} \chi)^{2}-\frac{1}{2} e^{\phi-12 A}(D b)^{2}-V\right)+\int 3 \chi \mathrm{d} \gamma_{\wedge} \mathrm{d} \gamma
\end{aligned}
$$

where we have defined,

$$
D b:=\mathrm{d} b+\frac{1}{2}\left(\xi_{A}^{\prime} \mathrm{d} \xi^{A}-\xi^{A} \mathrm{~d} \xi_{A}^{\prime}\right)+3 c_{0}(\gamma-\chi \alpha)+\Xi \alpha .
$$

Note that, for $c_{0} \neq 0,(3.16)$-(3.20) remain invariant under the shift (2.9).

\subsection{Comparison with $\mathscr{N}=2,4 \mathrm{~d}$ supergravity}

In terms of the fields introduced in (2.10), (2.11), and the Kähler potential (2.13), the action (3.19) takes the following form,

$$
\begin{aligned}
S_{4}=\int & \mathrm{d}^{4} x \sqrt{g_{4}}\left(R-2 \partial_{t} \partial_{\bar{t}} K_{V}|\mathrm{~d} t|^{2}-2 g_{a \bar{b}} \mathrm{~d} z^{a} \mathrm{~d} \bar{z}^{b}-2(\mathrm{~d} \hat{\phi})^{2}-\frac{1}{2} e^{4 \hat{\phi}}(D b)^{2}\right. \\
& +\frac{1}{2} e^{2 \hat{\phi}}\left(D \xi^{\prime}+\mathscr{M} \cdot D \xi\right) \cdot \mathfrak{I} \mathscr{M}^{-1} \cdot\left(D \xi^{\prime}+\mathscr{M}^{*} \cdot D \xi\right) \\
& \left.-\frac{1}{4} v^{3} \mathscr{F}^{2}-\frac{3}{4} v(\tilde{\mathscr{F}}+\chi \mathscr{F})^{2}-V\right)+\int 3 \chi \tilde{\mathscr{F}} \wedge \tilde{\mathscr{F}}+\chi^{3} \mathscr{F}_{\wedge} \mathscr{F}+3 \chi^{2} \mathscr{F}_{\wedge} \tilde{\mathscr{F}}
\end{aligned}
$$

It can be verified that (3.21) is of the form of $\mathscr{N}=2$ supergravity, given in (C.11). For the vector multiplet sector this was already shown in section 2. For the hypermultiplet sector we proceed as follows: the Killing vectors can be read off by comparing (3.9), (3.20) and (C.13),

$$
k_{I=0}^{u} \partial_{q^{u}}=-\Xi \partial_{b}-q_{A} \partial_{\xi_{A}^{\prime}}-p^{A} \partial_{\xi^{A}} ; \quad k_{I=1}^{u} \partial_{q^{u}}=-3 c_{0} \partial_{b},
$$


where $u, v=1, \ldots, 4 n_{H}$, and the $q^{u}$ coordinates are identified with $\hat{\phi}, b, \xi^{A}, \xi_{A}^{\prime}, \mathfrak{R} z^{a}, \mathfrak{I} z^{a}$. The hypermultiplet kinetic terms are manifestly of the form dictated by the quaternionic metric (C.12). Finally, to show that the potential (3.16) is of the from given in (C.14), we compute,

$$
h_{u v} k_{I}^{u} k_{J}^{v}=\frac{1}{2}\left(\begin{array}{cc}
e^{4 \hat{\phi}} \Xi^{2}-e^{2 \hat{\phi}}(q+\mathscr{M} \cdot p) \cdot \mathfrak{I} \mathscr{M}^{-1} \cdot\left(q+\mathscr{M}^{*} \cdot p\right) & -3 c_{0} e^{4 \hat{\phi}} \Xi \\
-3 c_{0} e^{4 \hat{\phi}} \Xi & 9 c_{0}^{2} e^{4 \hat{\phi}}
\end{array}\right),
$$

while (2.17), (2.18) remain unchanged. Substituting the latter together with (3.23) into (C.14) reproduces precisely the potential given in (3.16), in the absence of condensates: $\mathscr{A}=\mathscr{B}=0$.

\section{Positivity of the quartic gravitino condensate}

On ALE spaces one has the possibility to choose a gauge in which the spin connection is selfdual [55]. Covariantly-constant negative-chirality spinors in this gauge are just constant, and we may choose their basis as follows,

$$
\theta^{(1)}=\left(\begin{array}{l}
1 \\
0
\end{array}\right) ; \quad \theta^{(2)}=\left(\begin{array}{l}
0 \\
1
\end{array}\right)
$$

Let us now consider a spin-1 field, i.e. a field transforming in the three-dimensional irreducible representation of the $s u(2)$ algebra. This can be represented as a field with two symmetric spinor indices of the same chirality, $\phi_{\alpha \beta}=\phi_{\beta \alpha}$ (positive chirality) or $\phi^{\alpha \beta}=\phi^{\beta \alpha}$ (negative chirality). The Atiyah-Patodi-Singer theorem for a spin-1 field on an ALE space predicts that the number of positive-chirality zeromodes of the Dirac operator minus the number of negative-chirality zeromodes is equal to the Hirzebruch signature $(\tau)$ of the ALE space. Moreover it can be seen that there are no renormalizable negative-chirality zeromodes. It then follows that there are exactly $\tau$ spin-1 zeromodes of positive chirality.

By a similar argument as before, on ALE spaces there are exactly $2 \tau$ spin-3/2 (gravitino) zeromodes of positive chirality. They can be constructed as follows [56],

$$
\psi_{\mu \alpha}^{(i I)}=\phi_{\alpha \beta}^{(I)}\left(\tilde{\theta}^{(i)} \gamma_{\mu}\right)^{\beta} ; i=1,2 ; I=1, \ldots, \tau,
$$

where $\theta^{(i)}$ are covariantly-constant spinors of negative chirality, cf. (4.1), and $\phi_{\alpha \beta}^{(I)}$ are positivechirality spin-1 zeromodes.

Note that in this gauge the $\psi_{\mu}^{(i I)}$, s are automatically traceless, $\gamma^{\mu} \psi_{\mu}^{(i I)}=0$, as follows from,

$$
\left(C^{-1} \gamma^{\mu}\right)_{\gamma}{ }^{(\alpha}\left(C^{-1} \gamma_{\mu}\right)_{\delta}^{\beta)}=0
$$

Indeed if the above were not true, given an arbitrary spin-1 field of positive chirality $\phi$, one could construct a spin-1 field of negative chirality $\phi^{\prime}$, via $\phi^{\prime \gamma \delta}=\left(\gamma_{\mu}\right)^{\alpha \gamma}\left(\gamma^{\mu}\right)^{\beta \delta} \phi_{\alpha \beta}$. This is a contradiction, since the positive- and negative-chirality spin-1 representations transform under the $(\mathbf{3}, \mathbf{1})$ and $(\mathbf{1}, \mathbf{3})$ of $s o(4) \cong s u(2) \oplus s u(2)$, respectively.

In the path-integral over metrics approach, the leading contribution to the quartic-fermion condensate would come from spaces with four gravitino zero modes $(\tau=2)$. To a first approximation 
in the gravitational coupling, and up to a positive proportionality constant, it is obtained from the quartic terms in (2.5), by replacing each term by its expansion on the basis of zero modes (4.2),

$$
\psi_{\mu_{1}} \psi_{\mu_{2}} \psi_{\mu_{3}}^{\prime} \psi_{\mu_{4}}^{\prime} \rightarrow \sum_{i_{k}, I_{l}=1}^{2} \psi_{\mu_{1}}^{\left(i_{1} I_{1}\right)} \psi_{\mu_{2}}^{\left(i_{2} I_{2}\right)} \psi_{\mu_{3}}^{\left(i_{3} I_{3}\right)} \psi_{\mu_{4}}^{\left(i_{4} I_{4}\right)}
$$

where the sum is over all antisymmetrized permutations of the zero modes [56, 57].

We will now show that,

$$
\tilde{\psi}_{[\mu}^{(i I)} \psi_{v]}^{(j J)}-\tilde{\psi}_{[\mu}^{(j J)} \psi_{v]}^{(i I)}=0
$$

which implies that, within the present approximation, the first two terms on the right-hand side of the second line of (2.5) do not contribute to the quartic condensate, leaving as the only contribution the last positive-definite term. Indeed, using the form of the zero modes eq. (4.2) and the symmetry properties (A.9), we find,

$$
\tilde{\psi}_{[\mu}^{(i I)} \psi_{v]}^{(j J)}=\left(\tilde{\theta}^{(i)} \gamma_{[\mu}\right)^{\alpha}\left(\tilde{\theta}^{(j)} \gamma_{v]}\right)^{\beta}\left(\phi^{(I)} \cdot C^{-1} \phi^{(J)}\right)_{\alpha \beta}=\tilde{\psi}_{[\mu}^{(j J)} \psi_{v]}^{(i I)}
$$

Finally one can show that the last term in (2.5) gives,

$$
\mathscr{B} \propto \operatorname{tr}\left(\left(M^{(1)}\right)^{2}\right) \operatorname{tr}\left(\left(M^{(2)}\right)^{2}\right)-\left(\operatorname{tr}\left(M^{(1)} \cdot M^{(2)}\right)\right)^{2} \geq 0
$$

where we have defined the matrix $\left(M^{(I)}\right)^{\alpha}{ }_{\beta}:=C^{\alpha \delta} \phi_{\delta \beta}^{(I)}$, and we have taken into account (4.3), (A.10), (A.11), and the idenitites $\tilde{\boldsymbol{\theta}}^{(1)} \boldsymbol{\theta}^{(2)}=1, \tilde{\boldsymbol{\theta}}^{(1)} \boldsymbol{\theta}^{(1)}=\tilde{\boldsymbol{\theta}}^{(2)} \boldsymbol{\theta}^{(2)}=0$. Expression (4.7) is nonnegative by virtue of the Cauchy-Schwarz inequality.

\section{NK universal consistent truncation}

In this section we extend the consistent truncation of [30] to include the vectors $\alpha, \gamma$, the two-form $\beta$ and the additional scalar $\xi^{\prime}$, to complete the bosonic content of the universal sector of one gravity multiplet, one vector multiplet and one hypermultiplet of $\mathscr{N}=24 \mathrm{~d}$ supergravity. ${ }^{8}$ Explicitly we set,

$$
\begin{aligned}
& F=\mathrm{d} \alpha+m \beta+m \chi J ; \quad H=\mathrm{d} \chi_{\wedge} J-6 \omega \chi \operatorname{Re} \Omega+\mathrm{d} \beta \\
& G=\varphi \mathrm{vol}_{4}+\frac{1}{2}\left(m \chi^{2}+\xi\right) J_{\wedge} J+J_{\wedge}(\mathrm{d} \gamma-\alpha \wedge \mathrm{d} \chi+m \chi \beta)-\frac{1}{8 \omega} \mathrm{d} \xi_{\wedge} \operatorname{Im} \Omega-\frac{1}{2} D \xi^{\prime}{ }_{\wedge} \operatorname{Re} \Omega,
\end{aligned}
$$

where $\xi(x), \varphi(x)$ are real $4 \mathrm{~d}$ scalars. The covariant derivative is given by,

$$
D \xi^{\prime}:=\mathrm{d} \xi^{\prime}+12 \omega(\gamma-\chi \alpha)
$$

We have chosen to express $H$ in terms of the $4 \mathrm{~d}$ potential $\beta$ instead of the axion.

In the massive case, $m \neq 0$, the one-form $\alpha$ can be absorbed in ("eaten by") the two-form $\beta$ via the Stückelberg mechanism. This is however no longer true in the massless limit. In order to be able to treat both massive and massless cases on an equal footing, we keep both $\alpha$ and $\beta$ in the form ansatz. The massless limit is obtained by simply setting $m=0$ in (5.1).

\footnotetext{
${ }^{8}$ The scalar $\xi$ of the present paper was previously deonted by $\gamma$ in [30].
} 
Taking into account that for a NK manifold we have,

$$
\begin{aligned}
\mathrm{d} J & =-6 \omega \operatorname{Re} \Omega \\
\mathrm{d} \operatorname{Im} \Omega & =4 \omega J \wedge J,
\end{aligned}
$$

the ansatz (5.1) can be seen to automatically satisfy the Bianchi identities (B.7).

Our ansatz for the ten-dimensional metric reads,

$$
\mathrm{d} s_{(10)}^{2}=e^{2 A(x)}\left(e^{2 B(x)} g_{\mu \nu} \mathrm{d} x^{\mu} \mathrm{d} x^{v}+g_{m n} \mathrm{~d} y^{m} \mathrm{~d} y^{n}\right),
$$

where the scalars $A, B$ only depend on the four-dimensional coordinates $x^{\mu}$. This gives,

$$
\begin{aligned}
F_{m n}^{2} & =m^{2} \chi^{2} e^{-2 A} g_{m n} ; \quad F_{\mu \nu}^{2}=e^{-2 A-2 B}(\mathrm{~d} \alpha+m \beta)_{\mu \nu}^{2} \\
F^{2} & =e^{-4 A-4 B}(\mathrm{~d} \alpha+m \beta)^{2}+6 m^{2} \chi^{2} e^{-4 A} \\
H_{m n}^{2} & =2 e^{-4 A-2 B}(\partial \chi)^{2} g_{m n}+144 e^{-4 A} \omega^{2} \chi^{2} g_{m n} ; H_{\mu \nu}^{2}=6 e^{-4 A} \partial_{\mu} \chi \partial_{\nu} \chi+e^{-4 A-4 B} h_{\mu \nu}^{2} \\
H^{2} & =18 e^{-6 A-2 B}(\partial \chi)^{2}+e^{-6 A-6 B} h^{2}+864 e^{-6 A} \omega^{2} \chi^{2} \\
G_{m n}^{2} & =3 e^{-6 A-2 B}\left[\frac{1}{16 \omega^{2}}(\partial \xi)^{2}+\left(D \xi^{\prime}\right)^{2}\right] g_{m n}+12 e^{-6 A}\left(m \chi^{2}+\xi\right)^{2} g_{m n} \\
& +3 e^{-6 A-4 B}(\mathrm{~d} \gamma-\alpha \wedge \mathrm{d} \chi+m \chi \beta)^{2} g_{m n} \\
G_{\mu \nu}^{2} & =-6 e^{-6 A-6 B} \varphi^{2} g_{\mu \nu}+6 e^{-6 A}\left(\frac{1}{16 \omega^{2}} \partial_{\mu} \xi \partial_{\nu} \xi+D_{\mu} \xi^{\prime} D_{\nu} \xi^{\prime}\right) \\
& +18 e^{-6 A-2 B}(\mathrm{~d} \gamma-\alpha \wedge \mathrm{d} \chi+m \chi \beta)_{\mu \nu}^{2} \\
G^{2} & =-24 e^{-8 A-8 B} \varphi^{2}+24 e^{-8 A-2 B}\left[\frac{1}{16 \omega^{2}}(\partial \xi)^{2}+\left(D \xi^{\prime}\right)^{2}\right]+72\left(m \chi^{2}+\xi\right)^{2} e^{-8 A} \\
& +36 e^{-8 A-4 B}(\mathrm{~d} \gamma-\alpha \wedge \mathrm{d} \chi+m \chi \beta)^{2},
\end{aligned}
$$

where the contractions on the left-hand sides above are computed with respect to the ten-dimensional metric; the contractions on the right-hand sides are taken with respect to the unwarped metric. It is also useful to note the following expressions,

$$
\begin{aligned}
\star_{10} F= & \frac{1}{6} e^{6 A} \star_{4}(\mathrm{~d} \alpha+m \beta) J^{3}+\frac{1}{2} m \chi e^{6 A+4 B} \operatorname{vol}_{4 \wedge} J^{2} \\
\star_{10} H= & \frac{1}{2} e^{4 A+2 B} \star_{4} \mathrm{~d} \chi \jmath_{\wedge} J^{2}+\frac{1}{6} e^{4 A-2 B} \star_{4} h h^{3} J^{3}+6 \omega \chi e^{4 A+4 B} \operatorname{vol}_{4 \wedge} \operatorname{Im} \Omega \\
\star_{10} G= & -\frac{1}{6} \varphi e^{2 A-4 B} J^{3}+\left(m \chi^{2}+\xi\right) e^{2 A+4 B}{ }_{\operatorname{vol}_{4} \wedge} J+\frac{1}{2} e^{2 A} \star_{4}(\mathrm{~d} \gamma-\alpha \wedge \mathrm{d} \chi+m \chi \beta) \wedge J^{2} \\
& +\frac{1}{8 \omega} e^{2 A+2 B} \star_{4} \mathrm{~d} \xi_{\wedge} \operatorname{Re} \Omega-\frac{1}{2} e^{2 A+2 B} \star_{4} D \xi^{\prime}{ }_{\triangle} \operatorname{Im} \Omega,
\end{aligned}
$$

where the four-dimensional Hodge-star is taken with respect to the unwarped metric.

Plugging the above ansatz into the ten-dimensional EOM (B.4)-(B.6) we obtain the following: the internal $(m, n)$-components of the Einstein equations read,

$$
\begin{aligned}
20 e^{2 B} \omega^{2} & =e^{-8 A-2 B} \nabla^{\mu}\left(e^{8 A+2 B} \partial_{\mu} A\right)+\frac{1}{16} m^{2} e^{5 \phi / 2+2 A+2 B}+\frac{5}{16} e^{3 \phi / 2-2 A+2 B} m^{2} \chi^{2} \\
& -\frac{1}{32} e^{3 \phi / 2-2 A-2 B}(\mathrm{~d} \alpha+m \beta)^{2}+\frac{1}{8} e^{-\phi-4 A}(\partial \chi)^{2}-\frac{1}{48} e^{-\phi-4 A-4 B} h^{2}+18 e^{-\phi-4 A+2 B} \omega^{2} \chi^{2} \\
& -\frac{1}{32} e^{\phi / 2-6 A-2 B}(\mathrm{~d} \gamma-\alpha \wedge \mathrm{d} \chi+m \chi \beta)^{2}+\frac{1}{16} e^{\phi / 2-6 A}\left[\frac{1}{16 \omega^{2}}(\partial \xi)^{2}+\left(D \xi^{\prime}\right)^{2}\right] \\
& +\frac{3}{16} e^{\phi / 2-6 A-6 B} \varphi^{2}+\frac{7}{16} e^{\phi / 2-6 A+2 B}\left(m \chi^{2}+\xi\right)^{2} .
\end{aligned}
$$


The external $(\mu, v)$-components read,

$$
\begin{aligned}
R_{\mu \nu}^{(4)} & =g_{\mu \nu}\left(\nabla^{2} A+\nabla^{2} B+8(\partial A)^{2}+2(\partial B)^{2}+10 \partial A \cdot \partial B\right) \\
& -8 \partial_{\mu} A \partial_{\nu} A-2 \partial_{\mu} B \partial_{v} B-16 \partial_{(\mu} A \partial_{v} B+8 \nabla_{\mu} \partial_{v} A+2 \nabla_{\mu} \partial_{v} B \\
& +\frac{3}{2} e^{-\phi-4 A} \partial_{\mu} \chi \partial_{\nu} \chi+\frac{1}{2} e^{3 \phi / 2-2 A-2 B}(\mathrm{~d} \alpha+m \beta)_{\mu \nu}^{2}+\frac{1}{4} e^{\phi-4 A-4 B} h_{\mu \nu}^{2}+\frac{1}{2} \partial_{\mu} \phi \partial_{\nu} \phi \\
& +\frac{1}{2} e^{\phi / 2-6 A}\left(\frac{1}{16 \omega^{2}} \partial_{\mu} \xi \partial_{\nu} \xi+D_{\mu} \xi^{\prime} D_{\nu} \xi^{\prime}\right)+\frac{3}{2} e^{\phi / 2-6 A-2 B}(\mathrm{~d} \gamma-\alpha \wedge \mathrm{d} \chi+m \chi \beta)_{\mu \nu}^{2} \\
& +\frac{1}{16} g_{\mu \nu}\left(-\frac{1}{2} e^{3 \phi / 2-2 A-2 B}(\mathrm{~d} \alpha+m \beta)^{2}-\frac{1}{3} e^{\phi-4 A-4 B} h^{2}\right. \\
& -3 e^{\phi / 2-6 A}\left[\frac{1}{16 \omega^{2}}(\partial \xi)^{2}+\left(D \xi^{\prime}\right)^{2}\right]-6 e^{-\phi-4 A}(\partial \chi)^{2}-5 e^{\phi / 2-6 A-6 B} \varphi^{2} \\
& +m^{2} e^{5 \phi / 2+2 A+2 B}-3 m^{2} \chi^{2} e^{3 \phi / 2-2 A+2 B}-288 e^{-\phi-4 A+2 B} \omega^{2} \chi^{2} \\
& \left.-9\left(m \chi^{2}+\xi\right)^{2} e^{\phi / 2-6 A+2 B}-\frac{9}{2} e^{\phi / 2-6 A-2 B}(\mathrm{~d} \gamma-\alpha \wedge \mathrm{d} \chi+m \chi \beta)^{2}\right)
\end{aligned}
$$

while the mixed $(\mu, m)$-components are automatically satisfied. The dilaton equation reads,

$$
\begin{aligned}
0 & =e^{-10 A-4 B} \nabla^{\mu}\left(e^{8 A+2 B} \partial_{\mu} \phi\right)-\frac{1}{4} e^{\phi / 2-8 A-2 B}\left[\frac{1}{16 \omega^{2}}(\partial \xi)^{2}+\left(D \xi^{\prime}\right)^{2}\right] \\
& -\frac{3}{8} e^{3 \phi / 2-4 A-4 B}(\mathrm{~d} \alpha+m \beta)^{2}-\frac{5}{4} m^{2} e^{5 \phi / 2}-\frac{9}{4} e^{3 \phi / 2-4 A} m^{2} \chi^{2} \\
& +\frac{3}{2} e^{-\phi-6 A-2 B}(\partial \chi)^{2}+\frac{1}{12} e^{-\phi-6 A-6 B} h^{2}+72 e^{-\phi-6 A} \omega^{2} \chi^{2} \\
& +\frac{1}{4} e^{\phi / 2-8 A-8 B} \varphi^{2}-\frac{3}{4}\left(m \chi^{2}+\xi\right)^{2} e^{\phi / 2-8 A}-\frac{3}{8} e^{\phi / 2-8 A-4 B}(\mathrm{~d} \gamma-\alpha \wedge \mathrm{d} \chi+m \chi \beta)^{2} .
\end{aligned}
$$

The $F$-form equation of motion reduces to the condition,

$$
\begin{aligned}
\mathrm{d}\left(e^{3 \phi / 2+6 A} \star_{4}(\mathrm{~d} \alpha+m \beta)\right) & =\varphi e^{\phi / 2+2 A-4 B} \mathrm{~d} \beta-3 e^{\phi / 2+2 A} \mathrm{~d} \chi \wedge \star_{4}(\mathrm{~d} \gamma-\alpha \wedge \mathrm{d} \chi+m \chi \beta) \\
& -12 \omega \chi e^{\phi / 2+2 A+2 B} \star_{4} D \xi^{\prime} .
\end{aligned}
$$

The $H$-form equation reduces to the following two equations,

$$
\begin{aligned}
\mathrm{d}\left(e^{-\phi+4 A+2 B} \star_{4} \mathrm{~d} \chi\right) & =\left[\left(m \chi^{2}+\xi\right) \varphi-48 \omega^{2} e^{-\phi+4 A+4 B} \chi-2 m e^{\phi / 2+2 A+4 B}\left(m \chi^{2}+\xi\right) \chi\right. \\
& \left.-e^{3 \phi / 2+6 A+4 B} m^{2} \chi\right] \operatorname{vol}_{4}-e^{\phi / 2+2 A}(\mathrm{~d} \alpha+m \beta) \wedge \star_{4}(\mathrm{~d} \gamma-\alpha \wedge \mathrm{d} \chi+m \chi \beta) \\
& +(\mathrm{d} \gamma-\alpha \wedge \mathrm{d} \chi+m \chi \beta) \wedge(\mathrm{d} \gamma-\alpha \wedge \mathrm{d} \chi+m \chi \beta)
\end{aligned}
$$

and,

$$
\begin{aligned}
\mathrm{d}\left(e^{-\phi+4 A-2 B} \star_{4} \mathrm{~d} \beta\right) & =3\left(m \chi^{2}+\xi\right)(\mathrm{d} \gamma-\alpha \wedge \mathrm{d} \chi+m \chi \beta)-\frac{1}{4 \omega} \mathrm{d} \xi \wedge D \xi^{\prime}+e^{\phi / 2+2 A-4 B} \varphi(\mathrm{d} \alpha+m \beta) \\
& -e^{3 \phi / 2+6 A} m \star_{4}(\mathrm{~d} \alpha+m \beta)-3 e^{\phi / 2+2 A} m \chi \star_{4}(\mathrm{~d} \gamma-\alpha \wedge \mathrm{d} \chi+m \chi \beta)
\end{aligned}
$$


The $G$-form equation of motion reduces to,

$$
\begin{aligned}
\mathrm{d}\left(e^{\phi / 2+2 A+2 B} \star_{4} \mathrm{~d} \xi\right) & =4 \omega h_{\wedge} D \xi^{\prime}-48 \omega^{2}\left(e^{\phi / 2+2 A+4 B}\left(m \chi^{2}+\xi\right)-\chi \varphi\right) \operatorname{vol}_{4} \\
\mathrm{~d}\left(e^{\phi / 2+2 A+2 B} \star_{4} D \xi^{\prime}\right) & =-\frac{1}{4 \omega} h_{\wedge} \mathrm{d} \xi \\
\mathrm{d}\left(e^{\phi / 2+2 A} \star_{4}(\mathrm{~d} \gamma-\alpha \wedge \mathrm{d} \chi+m \chi \beta)\right) & =2 \mathrm{~d} \chi_{\wedge}(\mathrm{d} \gamma+m \chi \beta)+\left(m \chi^{2}+\xi\right) \mathrm{d} \beta+4 \omega e^{\phi / 2+2 A+2 B} \star_{4} D \xi^{\prime},
\end{aligned}
$$

together with the constraint,

$$
0=\mathrm{d}\left(\varphi e^{\phi / 2+2 A-4 B}+m \chi^{3}+3 \xi \chi\right)
$$

The latter can be integrated to solve for $\varphi$ in terms of the other fields,

$$
\varphi=\left(C-m \chi^{3}-3 \xi \chi\right) e^{-2 A+4 B-\phi / 2}
$$

where $C$ is an arbitrary constant.

\subsection{Action}

Setting $B=-4 A$, it can be seen that the dilaton and Einstein equations (5.7)-(5.9), can all be integrated to the following $4 \mathrm{~d}$ action, ${ }^{9}$

$$
\begin{aligned}
S_{4}= & \int \mathrm{d}^{4} x \sqrt{g}\left(R-24(\partial A)^{2}-\frac{1}{2}(\partial \phi)^{2}-\frac{3}{2} e^{-4 A-\phi}(\partial \chi)^{2}-\frac{1}{2} e^{-6 A+\phi / 2}\left[\frac{1}{16 \omega^{2}}(\partial \xi)^{2}+\left(D \xi^{\prime}\right)^{2}\right]\right. \\
& -\frac{1}{4} e^{3 \phi / 2+6 A}(\mathrm{~d} \alpha+m \beta)^{2}-\frac{3}{4} e^{\phi / 2+2 A}(\mathrm{~d} \gamma-\alpha \wedge \mathrm{d} \chi+m \chi \beta)^{2}-\frac{1}{12} e^{-\phi+12 A} \mathrm{~d} \beta^{2} \\
& -\frac{1}{2} m^{2} e^{-6 A+5 \phi / 2}-\frac{3}{2} m^{2} \chi^{2} e^{-10 A+3 \phi / 2}-72 \omega^{2} \chi^{2} e^{-12 A-\phi}-\frac{3}{2} e^{-14 A+\phi / 2}\left(\xi+m \chi^{2}\right)^{2} \\
& \left.-\frac{1}{2} e^{-\phi / 2-18 A}\left(C-m \chi^{3}-3 \xi \chi\right)^{2}+120 \omega^{2} e^{-8 A}\right)+S^{\mathrm{SC}}
\end{aligned}
$$

The Chern-Simons terms above, $S^{\mathrm{SC}}$, cannot be determined from (5.7)-(5.9): they must be reconstituted from the form equations of motion - which at the same time impose several additional consistency conditions on the action (5.16). We thus find that the form equations of motion are consistent with the Chern-Simons term,

$$
\begin{aligned}
\left.S^{\mathrm{SC}}=\int \beta_{\wedge}\left(-\frac{1}{4 \omega} \mathrm{d} \xi \wedge \mathrm{d} \xi^{\prime}+3 m \chi^{2} \mathrm{~d} \gamma+\mathrm{d}\left[\left(C-m \chi^{3}-3 \xi \chi\right) \alpha+3 \xi \gamma\right)\right]\right) \\
+m\left(\frac{1}{2} C+m \chi^{3}\right) \beta \wedge \beta+3 \chi \mathrm{d} \gamma \wedge \mathrm{d} \gamma
\end{aligned}
$$

\footnotetext{
${ }^{9}$ To obtain (5.16), the value of $\varphi$, as given in (5.15), must be substituted in (5.9) before integrating with respect to the dilaton. This action was first obtained in [35] by directly inserting the form ansatz into the 10d IIA theory, a procedure that does not in general lead to a CT. However it was shown that certain susy solutions of the 4d theory uplift to a well-known class of solutions [43, 44]. Ref. [35] also shows that the $4 \mathrm{~d}$ action is consistent with $\mathscr{N}=2$ gauged supergravity in its formulation with (massive) tensors [50, 51, 52].
} 


\subsection{Dualization}

To dualize the antisymmetric two-form potential $\beta$ to an axion $b$, which can only be performed in the massless limit $(m=0)$, we proceed as follows: first note that the second equation in (5.13) can be solved in order to express $D \xi^{\prime}$ in terms of a dual two-form $\tilde{\xi}^{\prime}$,

$$
e^{\phi / 2-6 A} \star_{4} D \xi^{\prime}=-\frac{1}{4 \omega} h \xi+\mathrm{d} \tilde{\xi}^{\prime} .
$$

Using the above, the last equation in (5.13) can be integrated to,

$$
e^{\phi / 2+2 A} \star_{4}(\mathrm{~d} \gamma-\alpha \wedge \mathrm{d} \chi)=2 \mathrm{~d} \chi_{\wedge} \gamma+4 \omega \tilde{\xi}^{\prime}+\mathrm{d} \tilde{\gamma},
$$

in terms of a dual one-form $\tilde{\gamma}$. Eq. (5.10) then integrates to,

$$
e^{3 \phi / 2+6 A} \star_{4} \mathrm{~d} \alpha=C \beta-12 \omega \chi \tilde{\xi}^{\prime}-3 \mathrm{~d} \chi_{\wedge} \tilde{\gamma},
$$

where we have also taken (5.15) into account. Substituting (5.18)-(5.20) into (5.12), we obtain the desired dualization condition,

$$
e^{-\phi+12 A} \star_{4} \mathrm{~d} \beta=\mathrm{d} b+\mathfrak{A},
$$

where we introduced the one-form,

$$
\mathfrak{A}:=\frac{1}{8 \omega}\left(\xi \mathrm{d} \xi^{\prime}-\xi^{\prime} \mathrm{d} \xi\right)+(C-3 \xi \chi) \alpha+3 \xi \gamma .
$$

Note that the dependence on $\tilde{\gamma}, \tilde{\xi}^{\prime}$ drops out of the $\beta$-dualization expression (5.21). In terms of the axion, the action reads,

$$
\begin{aligned}
S_{4}= & \int \mathrm{d}^{4} x \sqrt{g}\left(R-24(\partial A)^{2}-\frac{1}{2}(\partial \phi)^{2}-\frac{3}{2} e^{-4 A-\phi}(\partial \chi)^{2}-\frac{1}{2} e^{-6 A+\phi / 2}\left[\frac{1}{16 \omega^{2}}(\partial \xi)^{2}+\left(D \xi^{\prime}\right)^{2}\right]\right. \\
& -\frac{1}{4} e^{3 \phi / 2+6 A}(\mathrm{~d} \alpha)^{2}-\frac{3}{4} e^{\phi / 2+2 A}(\mathrm{~d} \gamma-\alpha \wedge \mathrm{d} \chi)^{2}-\frac{1}{12} e^{\phi-12 A}(\mathrm{~d} b+\mathfrak{A})^{2} \\
& \left.-72 \omega^{2} \chi^{2} e^{-12 A-\phi}-\frac{3}{2} e^{-14 A+\phi / 2} \xi^{2}-\frac{1}{2} e^{-\phi / 2-18 A}(C-3 \xi \chi)^{2}+120 \omega^{2} e^{-8 A}\right) \\
& +\int 3 \chi \mathrm{d} \gamma \wedge \mathrm{d} \gamma
\end{aligned}
$$

One can also make contact with the $\mathrm{CY}$ reduction of section 2 in the absence of condensates, by setting,

$$
\chi \rightarrow \chi-\frac{1}{12 \omega} b_{2} ; \quad \xi \rightarrow c_{0}+4 \omega \xi ; \quad b \rightarrow b-\frac{1}{8 \omega} c_{0} \xi^{\prime} ; \quad C \rightarrow-\frac{1}{4 \omega} b_{2} c_{0},
$$

and then taking the limit of vanishing torsion class: $\omega \rightarrow 0 .{ }^{10}$

\section{Discussion}

One of the motivations of constructing consistent truncations in the presence of condensates, is that it gives us a way of fixing the dependence of the condensates on the moduli. In the case considered here, the quadratic and quartic condensates decompose into a $4 \mathrm{~d}$ moduli-independent

\footnotetext{
${ }^{10}$ Compared to section 2, (5.24) corresponds to having a real background three-form flux $b_{0}$ (i.e. setting $b_{1}=0$ ). This can easily be achieved by shifting the phase of the CY three-form $\Omega$ by a suitable constant.
} 
piece (which we denoted by $\mathscr{A}$ and $\mathscr{B}$ respectively), and a moduli-dependent factor. For example, in the case of IIA compactified on a CY with $h^{2,1}=0$, the quadratic condensate is constrained by the consistency of the theory to enter the potential in the combination $v^{3 / 4} e^{-3 \hat{\phi} / 2} \mathscr{B}$, cf. (2.8), (2.10). I.e. imposing the consistency of the truncation determines the dependence of the condensates on the volume modulus and the $4 \mathrm{~d}$ dilaton.

It can be easily established that the effective theory of IIA compactified on a CY with $h^{2,1} \geq 1$ admits formal de Sitter solutions supported by a positive quartic gravitino condensate, just as in [31]. As in that reference, there are flat directions for the axion $b$ and the RR axions $\xi^{A}, \xi_{A}^{\prime}$, all of which are compact scalars. These solutions require a strictly positive quartic condensate, $\mathscr{B}>0$, but leave the quadratic condensate $\mathscr{A}$ unconstrained. In the presence of four spin- $3 / 2$ zero modes, a quartic condensate will be induced at one-loop order in the $4 \mathrm{~d}$ gravitational coupling. In the presence of two spin-3/2 zero modes, in which case $\mathscr{A} \neq 0, \mathscr{B}=0$ at one loop, a positive quartic condensate will plausibly be induced in the effective action at two loops in the gravitational coupling, $\mathscr{B} \propto \mathscr{A}^{2}$.

As we have seen in section $4, \mathscr{B}$ is non-negative in the background of ALE gravitational instantons with four spin-3/2 zero modes. However it is not clear whether such a condensate is non-zero. Imposing that the noncompact space should asymptote $\mathbb{R}^{4}$ at infinity, would exclude ALE gravitational instantons altogether: for the ALE instantons to contribute to the path integral, we must accept spacetimes with non-standard asymptotics. It is not clear whether this can make sense physically, ${ }^{11}$ although the fact that the Eguchi-Hanson ALE instanton [58] can be thought of as T-dual to a string theory solution with $\mathbb{R}^{4}$ asymptotics, would appear to support the admissibility of ALE instantons [59].

If we allow spacetimes with ALE asymptotics, for the de Sitter solutions of [31] and the present paper to go through, one needs not only non-vanishing condensates, but also non-vanishing background fluxes: $\left|b_{0}\right|, c_{0} \neq 0$. However, it has not been shown that ALE instanons can be embedded in a $4 \mathrm{~d}$ theory with such background fluxes. One way to address both of these issues would be to construct other gravitational instantons with $\mathbb{R}^{4}$ or $\mathrm{AdS}_{4}$ asymptotics ${ }^{12}$ in the presence of background fluxes. Such backgrounds would have non-vanishing profiles for the matter fields.

A CT may truncate out some of the "light" degrees of freedom. As a result, solutions obtained within the CT may be unstable when uplifted to the higher-dimensional theory. This phenomenon would not occur if the universal CT obtained in section 2 comes from compactification on a CY with $h^{1,1}=1, h^{2,1}=0$, since in this case all light degrees of freedom are already present in the CT. However, there is no known CY with such Hodge numbers to date, although there is no known theorem precluding its existence either. ${ }^{13}$ On the other hand, the effective action of section 3 should adequately capture all light degrees of freedom (at least below the energy scales set by the compactification and the background flux), but need not be a CT.

\footnotetext{
${ }^{11}$ In the case of the Eguchi-Hanson instanton, the discrete identifications are given by $(t, \vec{x}) \rightarrow-(t, \vec{x})$ at asymptotic infinity. To avoid closed timelike loops, upon Wick-rotation to Lorentzian signature, it would suffice to restrict to $t>0$, at the cost of rendering the space incomplete.

${ }^{12}$ As already mentioned, our derivation of the $4 \mathrm{~d}$ action (2.6), (3.12) is valid for condensates induced by any gravitational instantons with $4 \mathrm{~d}$ gravitino zero modes of positive chirality. In particular our results should also be applicable to the case of $\mathrm{K} 3$ instantons glued into asymptotic $\mathbb{R}^{4}$, as considered in [60].

${ }^{13}$ Note that such a CY, if it exists, cannot have a mirror.
} 
Naively relying on the $4 \mathrm{~d}$ effective action, in particular if the uplift is unknown, may lead to miss some crucial physics, see [61] for a recent example. A more important objection states that one should reject altogether solutions of the $4 \mathrm{~d}$ effective theory, such as de Sitter space, whose asymptotics are drastically different from the ones (flat Minkowski) used in deriving the 10d lowenergy string effective action, see [62] for a recent discussion. In other words, different asymptotic spacetime conditions should correspond to different quantum gravity models, and there is no a priori justification to using the low-energy effective action to extrapolate from one set of asymptotics to another. If this is true, string theory would currently have little to say about spacetimes with asymptotics other than flat or AdS space.

\section{Acknowledgment}

The present paper is an extended version of my talk at CORFU2019. I am grateful to the organizers of the conference for providing a pleasant and stimulating environment. I am also grateful to the physics department of TU Wien for kind hospitality. I would like to thank David Andriot, Niccolò Cribiori, Thomas Van Riet, Stefan Theisen and Timm Wrase for valuable discussions.

\section{A. Conventions}

We are following the "superspace conventions" where all forms are given by,

$$
\Phi_{(p)}=\frac{1}{p !} \Phi_{m_{1} \ldots m_{p}} \mathrm{~d} x^{m_{p}} \ldots \wedge \wedge \mathrm{d} x^{m_{1}} ; \mathrm{d}\left(\Phi_{(p)^{\wedge}} \Psi_{(q)}\right)=\Phi_{(p)^{\wedge}} \mathrm{d} \Psi_{(q)}+(-1)^{q} \mathrm{~d} \Phi_{(p)^{\wedge}} \Psi_{(q)} .
$$

The Hodge star of a $p$-form in $d$ dimensions is given by,

$$
\star\left(\mathrm{d} x^{a_{1}} \wedge \cdots \wedge \mathrm{d} x^{a_{p}}\right)=\frac{1}{(d-p) !} \varepsilon^{a_{1} \ldots a_{p}} b_{1 \ldots b_{d-p}} \mathrm{~d} x^{b_{1}} \wedge \cdots \wedge \mathrm{d} x^{b_{d-p}},
$$

so that for any spacetime signature,

$$
\Phi_{(p)} \wedge \star \Phi_{(p)}=\frac{1}{p !} \Phi^{2} \operatorname{vol}_{d}
$$

where we have defined,

$$
\operatorname{vol}_{d}:=\star 1 ; \quad \Phi^{2}:=\Phi_{m_{1} \ldots m_{p}} \Phi^{m_{1} \ldots m_{p}} .
$$

The following expressions are also useful,

$$
\begin{gathered}
\star_{6} \Omega=-i \Omega ; \quad \star_{6} \Phi^{(2,1)}=i \Phi^{(2,1)} . \\
\Omega \Omega^{*}=-\frac{i}{6}|\Omega|^{2} \operatorname{vol}_{6} ; \quad \Phi \wedge \Phi^{*}=\frac{i}{6}|\Phi|^{2} \operatorname{vol}_{6} ; \quad \operatorname{vol}_{6}=-\frac{1}{6} J^{3},
\end{gathered}
$$

where the Hodge star is with respect to the metric of the internal six-dimensional manifold. In the case of a CY, $\Omega, \Phi$ are harmonic, so their norms are constant (independent of the CY coordinates). From (A.6) it then follows,

$$
\int \Omega \Omega^{*}=-\frac{i}{6}|\Omega|^{2} V_{6} ; \quad \int \Phi \wedge \Phi^{*}=\frac{i}{6}|\Phi|^{2} V_{6}
$$


where $V_{6}:=\int \operatorname{vol}_{6}$ is constant, cf. the discussion below (3.2).

Our spinor conventions are as in [31]. A Euclidean $4 \mathrm{~d}$ Weyl spinor of positive, negative chirality is indicated with a lower, upper spinor index respectively: $\theta_{\alpha}, \chi^{\alpha}$, i.e. the position indicates the chirality. The $4 \mathrm{~d}$ gamma matrices, the charge conjugation and chirality matrices are decomposed into chiral blocks,

$$
\gamma_{\mu}=\left(\begin{array}{cc}
0 & \left(\gamma_{\mu}\right)_{\alpha \beta} \\
\left(\gamma_{\mu}\right)^{\alpha \beta} & 0
\end{array}\right) ; \quad C^{-1}=\left(\begin{array}{cc}
C^{\alpha \beta} & 0 \\
0 & C_{\alpha \beta}
\end{array}\right) ; \quad \gamma_{5}=\left(\begin{array}{cc}
\delta_{\alpha}^{\beta} & 0 \\
0 & -\delta^{\alpha}{ }_{\beta}
\end{array}\right) \text {. }
$$

The $\left(C^{-1} \gamma_{\mu_{1} \ldots \mu_{n}}\right)$ 's possess the following symmetry properties,

$$
\left(C^{-1} \gamma_{\mu}\right)_{\alpha}{ }^{\beta}=(-1)^{\frac{1}{2}(n-2)(n-3)}\left(C^{-1} \gamma_{\mu}\right)_{\alpha}^{\beta}
$$

The Fierz relation for two positive-chirality $4 \mathrm{~d}$ spinors reads,

$$
\theta_{\alpha} \chi_{\beta}=-\frac{1}{2}(\tilde{\theta} \chi) C_{\alpha \beta}-\frac{1}{8}\left(\tilde{\theta} \gamma_{\mu v} \chi\right)\left(\gamma^{\mu v} C\right)_{\alpha \beta},
$$

with a similar relation for negative-chirality spinors, and we have defined $\tilde{\theta}:=\theta^{\operatorname{Tr}} C^{-1}$. Let us also mention that for any two $4 \mathrm{~d}$ positive-chirality traceless vector-spinors, $\theta_{+}^{\mu}, \chi_{+}^{\mu}$, we have,

$$
\left(\theta_{+}^{\left[\mu_{1}\right.} \gamma^{\mu_{2} \mu_{3}} \chi_{+}^{\left.\mu_{4}\right]}\right)=\frac{1}{12} \varepsilon^{\mu_{1} \mu_{2} \mu_{3} \mu_{4}}\left(\theta_{+}^{\lambda} \chi_{\rho+}\right)
$$

\section{B. The 10d theory}

In the conventions of [30], upon setting the dilatino to zero, the IIA action reads,

$$
\begin{aligned}
S=S_{b} & +\frac{1}{2 \kappa_{10}^{2}} \int \mathrm{d}^{10} x \sqrt{g}\left\{2\left(\tilde{\Psi}_{M} \Gamma^{M N P} \nabla_{N} \Psi_{P}\right)+\frac{1}{2} e^{5 \phi / 4} m\left(\tilde{\Psi}_{M} \Gamma^{M N} \Psi_{N}\right)\right. \\
& -\frac{1}{2 \cdot 2 !} e^{3 \phi / 4} F_{M_{1} M_{2}}\left(\tilde{\Psi}^{M} \Gamma_{[M} \Gamma^{M_{1} M_{2}} \Gamma_{N]} \Gamma_{11} \Psi^{N}\right) \\
& -\frac{1}{2 \cdot 3 !} e^{-\phi / 2} H_{M_{1} \ldots M_{3}}\left(\tilde{\Psi}^{M} \Gamma_{[M} \Gamma^{M_{1} \ldots M_{3}} \Gamma_{N]} \Gamma_{11} \Psi^{N}\right) \\
& \left.+\frac{1}{2 \cdot 4 !} e^{\phi / 4} G_{M_{1} \ldots M_{4}}\left(\tilde{\Psi}^{M} \Gamma_{[M} \Gamma^{M_{1} \ldots M_{4}} \Gamma_{N]} \Psi^{N}\right)+L_{\Psi^{4}}\right\}
\end{aligned}
$$

where $\Psi_{M}$ is the gravitino; $S_{b}$ denotes the bosonic sector,

$$
\begin{aligned}
S_{b}=\frac{1}{2 \kappa_{10}^{2}} \int \mathrm{d}^{10} x \sqrt{g}( & -R+\frac{1}{2}(\partial \phi)^{2}+\frac{1}{2 \cdot 2 !} e^{3 \phi / 2} F^{2} \\
& \left.+\frac{1}{2 \cdot 3 !} e^{-\phi} H^{2}+\frac{1}{2 \cdot 4 !} e^{\phi / 2} G^{2}+\frac{1}{2} m^{2} e^{5 \phi / 2}\right)+S^{\mathrm{CS}},
\end{aligned}
$$

and $S^{\mathrm{CS}}$ is the Chern-Simons term, which in the massless limit is simplifies to,

$$
S^{\mathrm{CS}}=\int\left(\frac{1}{2} B_{\wedge} G^{2}-\frac{1}{2} F_{\wedge} B^{2}{ }_{\wedge} G+\frac{1}{6} F^{2}{ }_{\wedge} B^{3}\right) .
$$

$L_{\Psi^{4}}$ in (B.1) denotes the 24 quartic gravitino terms given in [63]. Of these only four can have a nonvanishing VEV in a gravitational instanton background with positive-chirality $4 \mathrm{~d}$ zero modes, resulting in the expression given in (2.5). 
We emphasize that the action (B.1) should be regarded as a book-keeping device whose variation with respect to the bosonic fields gives the correct bosonic equations of motion in the presence of gravitino condensates. Furthermore, the fermionic equations of motion are trivially satisfied in the maximally-symmetric vacuum. The (bosonic) equations of motion (EOM) following from (B.1) are as follows:

Dilaton EOM,

$$
\begin{aligned}
0 & =-\nabla^{2} \phi+\frac{3}{8} e^{3 \phi / 2} F^{2}-\frac{1}{12} e^{-\phi} H^{2}+\frac{1}{96} e^{\phi / 2} G^{2}+\frac{5}{4} m^{2} e^{5 \phi / 2} \\
& +\frac{5}{8} e^{5 \phi / 4} m\left(\tilde{\Psi}_{M} \Gamma^{M N} \Psi_{N}\right) \\
& -\frac{3}{16} e^{3 \phi / 4} F_{M_{1} M_{2}}\left(\tilde{\Psi}^{M} \Gamma_{[M} \Gamma^{M_{1} M_{2}} \Gamma_{N]} \Gamma_{11} \Psi^{N}\right) \\
& +\frac{1}{24} e^{-\phi / 2} H_{M_{1} \ldots M_{3}}\left(\tilde{\Psi}^{M} \Gamma_{[M} \Gamma^{M_{1} \ldots M_{3}} \Gamma_{N]} \Gamma_{11} \Psi^{N}\right) \\
& +\frac{1}{192} e^{\phi / 4} G_{M_{1} \ldots M_{4}}\left(\tilde{\Psi}^{M} \Gamma_{[M} \Gamma^{M_{1} \ldots M_{4}} \Gamma_{N]} \Psi^{N}\right)
\end{aligned}
$$

Einstein EOM,

$$
\begin{aligned}
& R_{M N}=\frac{1}{2} \partial_{M} \phi \partial_{N} \phi+\frac{1}{16} m^{2} e^{5 \phi / 2} g_{M N}+\frac{1}{4} e^{3 \phi / 2}\left(2 F_{M N}^{2}-\frac{1}{8} g_{M N} F^{2}\right) \\
& +\frac{1}{12} e^{-\phi}\left(3 H_{M N}^{2}-\frac{1}{4} g_{M N} H^{2}\right)+\frac{1}{48} e^{\phi / 2}\left(4 G_{M N}^{2}-\frac{3}{8} g_{M N} G^{2}\right) \\
& +\frac{1}{24} e^{\phi / 4} G_{(M \mid}{ }^{M_{1} M_{2} M_{3}}\left(\tilde{\Psi}_{P} \Gamma^{[P} \Gamma_{\mid N) M_{1} M_{2} M_{3}} \Gamma^{Q]} \Psi_{Q}\right) \\
& -\frac{1}{96} e^{\phi / 4} G_{M_{1} \ldots M_{4}}\left\{\left(\tilde{\Psi}_{P} \Gamma_{(M} \Gamma^{M_{1} \ldots M_{4}} \Gamma^{P} \Psi_{N)}\right)-\left(\tilde{\Psi}_{P} \Gamma^{P} \Gamma^{M_{1} \ldots M_{4}} \Gamma_{(M} \Psi_{N)}\right)\right. \\
& \left.+\frac{1}{2} g_{M N}\left(\tilde{\Psi}^{P} \Gamma_{[P} \Gamma^{M_{1} \ldots M_{4}} \Gamma_{Q]} \Psi^{Q}\right)\right\}-\frac{1}{8} g_{M N} L_{\Psi^{4}}+\frac{\delta L_{\Psi^{4}}}{\delta g^{M N}},
\end{aligned}
$$

where we have set: $\Phi_{M N}^{2}:=\Phi_{M M_{2} \ldots M_{p}} \Phi_{N}{ }^{M_{2} \ldots M_{p}}$, for any $p$-form $\Phi$. In the Einstein equation above we have not included the gravitino couplings to the two- and three-forms, which vanish in the background given by (2.4).

Form EOM's,

$$
\begin{aligned}
0 & =\mathrm{d} \star\left[e^{3 \phi / 2} F-\frac{1}{2} e^{3 \phi / 4}\left(\tilde{\Psi}^{M} \Gamma_{[M} \Gamma^{(2)} \Gamma_{N]} \Gamma_{11} \Psi^{N}\right)\right]+H \wedge \star\left[e^{\phi / 2} G+\frac{1}{2} e^{\phi / 4}\left(\tilde{\Psi}^{M} \Gamma_{[M} \Gamma^{(4)} \Gamma_{N]} \Psi^{N}\right)\right] \\
0 & \left.=\mathrm{d} \star\left[e^{-\phi} H-\frac{1}{2} e^{-\phi / 2}\left(\tilde{\Psi}^{M} \Gamma_{[M} \Gamma^{(3)} \Gamma_{N]} \Gamma_{11} \Psi^{N}\right)\right]+e^{\phi / 2} F_{\wedge} \star e^{\phi / 2} G+\frac{1}{2} e^{\phi / 4}\left(\tilde{\Psi}^{M} \Gamma_{[M} \Gamma^{(4)} \Gamma_{N]} \Psi^{N}\right)\right] \\
& -\frac{1}{2} G \wedge G+m \star\left[e^{3 \phi / 2} F-\frac{1}{2} e^{3 \phi / 4}\left(\tilde{\Psi}^{M} \Gamma_{[M} \Gamma^{(2)} \Gamma_{N]} \Gamma_{11} \Psi^{N}\right)\right] \\
0 & =\mathrm{d} \star\left[e^{\phi / 2} G+\frac{1}{2} e^{\phi / 4}\left(\tilde{\Psi}^{M} \Gamma_{[M} \Gamma^{(4)} \Gamma_{N]} \Psi^{N}\right)\right]-H \wedge G
\end{aligned}
$$

where $\Gamma^{(p)}:=\frac{1}{p !} \Gamma_{M_{1} \ldots M_{p}} \mathrm{~d} x^{M_{p}} \wedge \cdots \wedge \mathrm{d} x^{M_{1}}$. In addition the forms obey the Bianchi identities,

$$
\mathrm{d} F=m H ; \quad \mathrm{d} H=0 ; \mathrm{d} G=H \wedge F .
$$




\section{Gauged $\mathscr{N}=2,4 \mathrm{~d}$ supergravity}

Massless IIA compactification with background fluxes on a CY, $Y$, with Hodge numbers $\left(h^{1,1}, h^{2,1}\right)$, results in gauged $\mathscr{N}=2$ supergravity with $n_{V}=h^{1,1}$ vector multiplets and $n_{H}=h^{2,1}+1$ hypermultiplets. The vector multiplets contain $n_{V}$ complex scalars $t^{i}, i=1, \ldots, n_{V}$, arising from the expansion of the complexified Kähler form,

$$
B+i J=t^{i} \omega_{i}
$$

on a basis $\left\{\omega_{i}\right\}$ of real harmonic $(1,1)$-forms of the CY. Let us introduce the following definitions,

$$
\mathscr{K}=\frac{1}{6} \int_{Y} J_{\wedge} J_{\wedge} J ; \quad \mathscr{K}_{i}=\int_{Y} \omega_{i \wedge} J_{\wedge} J ; \quad \mathscr{K}_{i j}=\int_{Y} \omega_{i \wedge} \omega_{j \wedge} J ; \quad \mathscr{K}_{i j k}=\int_{Y} \omega_{i \wedge} \omega_{j^{\wedge}} \omega_{k} .
$$

The associated Kähler potential is then given by,

$$
e^{-K_{V}}=8 \mathscr{K}
$$

We will also need the symmetric matrix $\mathscr{N}_{I J}, I, J=0,1, \ldots, n_{V}$, whose components can be expressed as follows,

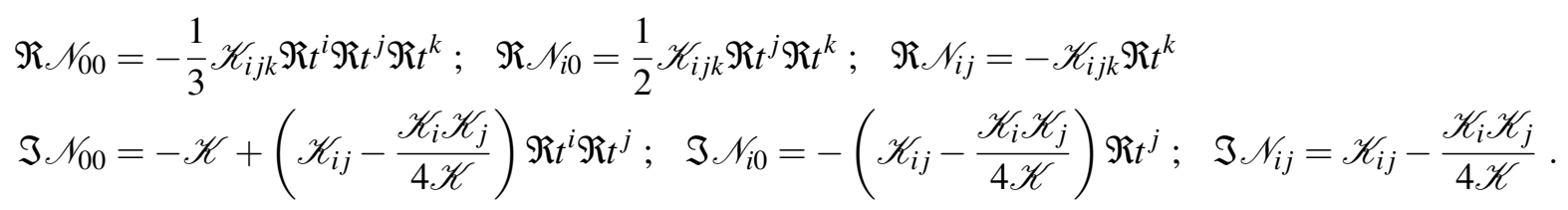

In order to describe the hypermultiplets it is useful to introduce a real basis $\left\{\alpha_{A}, \beta^{B}\right\}$ of harmonic three-forms on $Y$ normalized as follows,

$$
\int_{Y} \alpha_{A \wedge} \beta^{B}=\delta_{A}^{B} ; \quad \int_{Y} \alpha_{A \wedge} \alpha_{B}=\int_{Y} \beta^{A} \beta^{B}=0
$$

for $A, B=0,1, \ldots, h^{2,1}$. The periods of $\Omega$ are then given by,

$$
\Omega=Z^{A} \alpha_{A}-\mathscr{G}_{A} \beta^{A}
$$

where $\mathscr{G}_{A}=\partial \mathscr{G} / \partial Z^{A}$, with $\mathscr{G}(Z)$ a holomorphic, homogeneous, degree-two function of the projective coordinates $Z^{A}$. The complex structure deformations are parameterized by the affine coordinates $z^{a}=Z^{a} / Z^{0}$, with $a=1, \ldots, h^{2,1}$. This is equivalent to setting $Z^{A}=\left(1, z^{a}\right)$. The metric on the space of complex structure deformations $g_{a \bar{b}}=\partial_{z^{a}} \partial_{\bar{z}^{b}} K_{H}$ is associated with the Kähler potential,

$$
K_{H}=-\ln \left(i \int_{Y} \Omega \bar{\Omega}\right)=-\ln i\left(\bar{Z}^{A} \mathscr{G}_{A}-Z^{A} \overline{\mathscr{G}}_{A}\right) .
$$

We will also need to define the symmetric matrix $\mathscr{M}$,

$$
\mathscr{M}_{A B}:=\overline{\mathscr{G}}_{A B}+2 i \frac{(\mathfrak{I} \mathscr{G})_{A C} Z^{C}(\mathfrak{I} \mathscr{G})_{B D} Z^{D}}{Z^{C}(\mathfrak{I} \mathscr{G})_{C D} Z^{D}},
$$

where $\mathscr{G}_{A B}:=\partial^{2} \mathscr{G} / \partial Z^{A} \partial Z^{B}$. 
The matrix $\mathscr{M}_{A B}$ can be used to express the Hodge dual of the three-form basis,

$$
{ }_{\star_{6}} \alpha_{A}=A_{A}{ }^{B} \alpha_{B}+B_{A B} \beta^{B} ; \quad \star_{6} \beta^{A}=C^{A B} \alpha_{B}-\beta^{B} A_{B}{ }^{A},
$$

where $A_{A}{ }^{B}, B_{A B}, C^{A B}$ are given by,

$$
A=\mathfrak{R} \mathscr{M} \cdot \mathfrak{I} \mathscr{M}^{-1} ; \quad B=-\mathfrak{I} \mathscr{M}-\mathfrak{R} \mathscr{M} \cdot \mathfrak{I} \mathscr{M}^{-1} \cdot \mathfrak{R} \mathscr{M} ; \quad C=\mathfrak{I} \mathscr{M}^{-1},
$$

and we have used matrix notation.

We shall be interested in the bosonic sector of $\mathscr{N}=2$ supergravity with $n_{V}$ ungauged vector multiplets and $n_{H}$ gauged hypermultiplets,

$$
S_{4}=\int \mathrm{d}^{4} x \sqrt{g_{4}}\left(R-2 g_{i j} \partial_{\mu} t^{i} \partial^{\mu} \bar{t}^{j}-2 h_{u v} D_{\mu} q^{u} D^{\mu} q^{v}+\mathfrak{I} \mathscr{N}_{I J} F_{\mu v}^{I} F^{J \mu \nu}-V\right)+\int \Re \mathscr{N}_{I J} F^{I}{ }_{\wedge} F^{J},
$$

where $u, v=1, \ldots, 4 n_{H}$. The metric $h_{u v}$ reads,

$$
\begin{aligned}
h_{u v} D q^{u} D q^{v} & =(\mathrm{d} \hat{\phi})^{2}+\frac{1}{4} e^{4 \hat{\phi}}(D b)^{2}+g_{a \bar{b}} \mathrm{~d} z^{a} \mathrm{~d} \bar{z}^{b} \\
& -\frac{1}{4} e^{2 \hat{\phi}}\left(\mathfrak{I} \mathscr{M}^{-1}\right)^{A B}\left(D \xi_{A}^{\prime}+\mathscr{M}_{A C} \mathrm{~d} \xi^{C}\right)\left(D \xi_{B}^{\prime}+\mathscr{M}_{B D}^{*} \mathrm{~d} \xi^{D}\right),
\end{aligned}
$$

where the covariant derivatives,

$$
D q^{u}=\mathrm{d} q^{u}-k_{I}^{u} A^{I}
$$

with $\mathrm{d} A^{I}=F^{I}$, are given in terms of the Killing vectors $k_{I}^{u}$. The potential of the theory is expressed in terms of the latter as follows,

$$
V=8 e^{K} X^{I} \bar{X}^{J} h_{u v} k_{I}^{u} k_{J}^{v}-\left(8 e^{K} X^{I} \bar{X}^{J}+\left(\mathfrak{I} \mathscr{N}^{-1}\right)^{I J}\right) \vec{P}_{I} \cdot \vec{P}_{J}
$$

where the $X^{I}$ are holomorphic functions of the complexified Kähler coordinates, and may be chosen as $X^{I}=\left(1, t^{i}\right)$. The prepotentials $\vec{P}_{I}$ is given as follows: the metric $h_{u v}$ is quaternionic and possesses an associated $S U(2)$ connection $\vec{\omega}=\left(\omega^{1}, \omega^{2}, \omega^{3}\right)$,

$$
\begin{aligned}
& \omega^{1}=-\frac{1}{\sqrt{2}} e^{\frac{1}{2} K_{H}+\hat{\phi}} Z^{A}\left(\mathrm{~d} \xi_{A}^{\prime}+\mathscr{M}_{A B} \mathrm{~d} \xi^{B}\right)+\text { c.c. } \\
& \omega^{2}=\frac{1}{\sqrt{2}} i e^{\frac{1}{2} K_{H}+\hat{\phi}} Z^{A}\left(\mathrm{~d} \xi_{A}^{\prime}+\mathscr{M}_{A B} \mathrm{~d} \xi^{B}\right)+\text { c.c. } \\
& \omega^{3}=\frac{1}{\sqrt{2}} e^{2 \hat{\phi}}\left(\mathrm{d} b+\frac{1}{2}\left(\xi^{A} \mathrm{~d} \xi_{A}^{\prime}-\xi_{A}^{\prime} \mathrm{d} \xi^{A}\right)\right)+\ldots
\end{aligned}
$$

where the ellipses denote the directions along $\mathrm{d} z^{a}$. We then have [49],

$$
\vec{P}_{I}=k_{I}^{u} \vec{\omega}_{u}
$$

\section{References}

[1] B. de Wit and H. Nicolai. The Consistency of the $S^{7}$ Truncation in D=11 Supergravity. Nucl. Phys., B281:211-240, 1987.

[2] Horatiu Nastase, Diana Vaman, and Peter van Nieuwenhuizen. Consistent nonlinear KK reduction of 11d supergravity on $\mathrm{AdS}_{7} \times \mathrm{S}^{4}$ and selfduality in odd dimensions. Phys. Lett., B469:96-102, 1999. 
[3] Horatiu Nastase, Diana Vaman, and Peter van Nieuwenhuizen. Consistency of the $\mathrm{AdS}_{7} \times \mathrm{S}^{4}$ reduction and the origin of selfduality in odd dimensions. Nucl. Phys., B581:179-239, 2000.

[4] Hong Lu, C. N. Pope, and Tuan A. Tran. Five-dimensional N=4, SU(2) $\times U(1)$ gauged supergravity from type IIB. Phys. Lett., B475:261-268, 2000.

[5] Mirjam Cvetic, Hong Lu, C. N. Pope, A. Sadrzadeh, and Tuan A. Tran. Consistent SO(6) reduction of type IIB supergravity on $\mathrm{S}^{5}$. Nucl. Phys., B586:275-286, 2000.

[6] Adolfo Guarino, Daniel L. Jafferis, and Oscar Varela. String Theory Origin of Dyonic N=8 Supergravity and Its Chern-Simons Duals. Phys. Rev. Lett., 115(9):091601, 2015.

[7] Folkert Mueller-Hoissen and Richard Stuckl. Coset Spaces and Ten-dimensional Unified Theories. Class. Quant. Grav., 5:27, 1988.

[8] D. Kapetanakis and G. Zoupanos. Coset space dimensional reduction of gauge theories. Phys. Rept., 219:4-76, 1992.

[9] Iosif Bena, Gregory Giecold, Mariana Grana, Nick Halmagyi, and Francesco Orsi. Supersymmetric Consistent Truncations of IIB on $T^{1,1}$. JHEP, 04:021, 2011.

[10] Davide Cassani and Paul Koerber. Tri-Sasakian consistent reduction. JHEP, 01:086, 2012.

[11] Davide Cassani, Paul Koerber, and Oscar Varela. All homogeneous N=2 M-theory truncations with supersymmetric $\mathrm{AdS}_{4}$ vacua. JHEP, 11:173, 2012.

[12] Jerome P. Gauntlett and Oscar Varela. Consistent Kaluza-Klein reductions for general supersymmetric AdS solutions. Phys. Rev., D76:126007, 2007.

[13] Davide Cassani and Amir-Kian Kashani-Poor. Exploiting N=2 in consistent coset reductions of type IIA. Nucl. Phys., B817:25-57, 2009.

[14] Davide Cassani, Gianguido Dall'Agata, and Anton F. Faedo. Type IIB supergravity on squashed Sasaki-Einstein manifolds. JHEP, 05:094, 2010.

[15] Kostas Skenderis, Marika Taylor, and Dimitrios Tsimpis. A Consistent truncation of IIB supergravity on manifolds admitting a Sasaki-Einstein structure. JHEP, 06:025, 2010.

[16] Jerome P. Gauntlett and Oscar Varela. Universal Kaluza-Klein reductions of type IIB to N=4 supergravity in five dimensions. JHEP, 06:081, 2010.

[17] James T. Liu, Phillip Szepietowski, and Zhichen Zhao. Consistent massive truncations of IIB supergravity on Sasaki-Einstein manifolds. Phys. Rev., D81:124028, 2010.

[18] Gerardo Aldazabal, Walter Baron, Diego Marques, and Carmen Nunez. The effective action of Double Field Theory. JHEP, 11:052, 2011. [Erratum: JHEP11,109(2011)].

[19] David Geissbuhler. Double Field Theory and N=4 Gauged Supergravity. JHEP, 11:116, 2011.

[20] Kanghoon Lee, Charles Strickland-Constable, and Daniel Waldram. Spheres, generalised parallelisability and consistent truncations. Fortsch. Phys., 65(10-11):1700048, 2017.

[21] Olaf Hohm and Henning Samtleben. Consistent Kaluza-Klein Truncations via Exceptional Field Theory. JHEP, 01:131, 2015.

[22] Davide Cassani, Oscar de Felice, Michela Petrini, Charles Strickland-Constable, and Daniel Waldram. Exceptional generalised geometry for massive IIA and consistent reductions. JHEP, 08:074, 2016.

[23] Arnaud Baguet, Olaf Hohm, and Henning Samtleben. Consistent Type IIB Reductions to Maximal 5D Supergravity. Phys. Rev., D92(6):065004, 2015. 
[24] A. Baguet, C. N. Pope, and H. Samtleben. Consistent Pauli reduction on group manifolds. Phys. Lett., B752:278-284, 2016.

[25] Franz Ciceri, Adolfo Guarino, and Gianluca Inverso. The exceptional story of massive IIA supergravity. JHEP, 08:154, 2016.

[26] Gianluca Inverso, Henning Samtleben, and Mario Trigiante. Type II supergravity origin of dyonic gaugings. Phys. Rev., D95(6):066020, 2017.

[27] Gianluca Inverso. Generalised Scherk-Schwarz reductions from gauged supergravity. JHEP, 12:124, 2017.

[28] Emanuel Malek. Half-Maximal Supersymmetry from Exceptional Field Theory. Fortsch. Phys., 65(10-11):1700061, 2017.

[29] Emanuel Malek, Henning Samtleben, and Valentí Vall Camell. Supersymmetric AdS 7 and AdS 6 vacua and their minimal consistent truncations from exceptional field theory. Phys. Lett., B786:171-179, 2018.

[30] Robin Terrisse and Dimitrios Tsimpis. Consistent truncation with dilatino condensation on nearly Kähler and Calabi-Yau manifolds. JHEP, 02:088, 2019.

[31] Robin Terrisse and Dimitrios Tsimpis. Consistent truncation and de Sitter space from gravitational instantons. JHEP, 07:034, 2019.

[32] J.-B. Butruille. Homogeneous nearly Kähler manifolds. ArXiv e-prints math/0612655, December 2006.

[33] Lorenzo Foscolo and Mark Haskins. New G2 holonomy cones and exotic nearly Kaehler structures on the 6-sphere and the product of a pair of 3-spheres. Annals Math., 185:59-130, 2017.

[34] A. Spiro and F. Podestá. Six-dimensional nearly Kaehler manifolds of cohomogeneity one (II). ArXiv e-prints math.DG/1011.4681, November 2010.

[35] Amir-Kian Kashani-Poor. Nearly Kaehler Reduction. JHEP, 11:026, 2007.

[36] Sebastien Gurrieri, Jan Louis, Andrei Micu, and Daniel Waldram. Mirror symmetry in generalized Calabi-Yau compactifications. Nucl. Phys., B654:61-113, 2003.

[37] R. D'Auria, S. Ferrara, M. Trigiante, and S. Vaula. Gauging the Heisenberg algebra of special quaternionic manifolds. Phys. Lett., B610:147-151, 2005.

[38] Thomas House and Eran Palti. Effective action of (massive) IIA on manifolds with SU(3) structure. Phys. Rev., D72:026004, 2005.

[39] Mariana Grana, Jan Louis, and Daniel Waldram. Hitchin functionals in $\mathrm{N}=2$ supergravity. JHEP, 01:008, 2006.

[40] Jan Louis and Andrei Micu. Heterotic-Type IIA duality with fluxes. JHEP, 03:026, 2007.

[41] Amir-Kian Kashani-Poor and Ruben Minasian. Towards reduction of type II theories on SU(3) structure manifolds. JHEP, 03:109, 2007.

[42] David Andriot and Dimitrios Tsimpis. Laplacian spectrum on a nilmanifold, truncations and effective theories. JHEP, 09:096, 2018.

[43] Klaus Behrndt and Mirjam Cvetic. General N = 1 supersymmetric flux vacua of (massive) type IIA string theory. Phys. Rev. Lett., 95:021601, 2005. 
[44] Dieter Lüst and Dimitrios Tsimpis. Supersymmetric $\mathrm{AdS}_{4}$ compactifications of IIA supergravity. JHEP, 02:027, 2005.

[45] Adolfo Guarino and Oscar Varela. Consistent $\mathscr{N}=8$ truncation of massive IIA on $\mathrm{S}^{6}$. JHEP, 12:020, 2015.

[46] S. Ferrara and S. Sabharwal. Quaternionic Manifolds for Type II Superstring Vacua of Calabi-Yau Spaces. Nucl. Phys., B332:317-332, 1990.

[47] M. Bodner, A. C. Cadavid, and S. Ferrara. (2,2) vacuum configurations for type IIA superstrings: N=2 supergravity Lagrangians and algebraic geometry. Class. Quant. Grav., 8:789-808, 1991.

[48] Jan Louis and Andrei Micu. Type 2 theories compactified on Calabi-Yau threefolds in the presence of background fluxes. Nucl. Phys., B635:395-431, 2002.

[49] Jeremy Michelson. Compactifications of type IIB strings to four-dimensions with nontrivial classical potential. Nucl. Phys., B495:127-148, 1997.

[50] Ulrich Theis and Stefan Vandoren. N=2 supersymmetric scalar tensor couplings. JHEP, 04:042, 2003.

[51] Gianguido Dall'Agata, Riccardo D'Auria, L. Sommovigo, and Silvia Vaula. D = 4, N=2 gauged supergravity in the presence of tensor multiplets. Nucl. Phys., B682:243-264, 2004.

[52] Riccardo D'Auria, L. Sommovigo, and Silvia Vaula. $\mathrm{N}=2$ supergravity Lagrangian coupled to tensor multiplets with electric and magnetic fluxes. JHEP, 11:028, 2004.

[53] Steven B. Giddings and Andrew Strominger. Axion Induced Topology Change in Quantum Gravity and String Theory. Nucl. Phys., B306:890-907, 1988.

[54] Andrew Strominger. Special Geometry. Commun. Math. Phys., 133:163-180, 1990.

[55] Tohru Eguchi, Peter B. Gilkey, and Andrew J. Hanson. Gravitation, Gauge Theories and Differential Geometry. Phys. Rept., 66:213, 1980.

[56] S. W. Hawking and C. N. Pope. Symmetry Breaking by Instantons in Supergravity. Nucl. Phys., B146:381-392, 1978.

[57] K. Konishi, N. Magnoli, and H. Panagopoulos. Spontaneous Breaking of Local Supersymmetry by Gravitational Instantons. Nucl. Phys., B309:201, 1988.

[58] Tohru Eguchi and Andrew J. Hanson. Selfdual Solutions to Euclidean Gravity. Annals Phys., 120:82, 1979.

[59] M. Bianchi, F. Fucito, G. C. Rossi, and M. Martellini. ALE instantons in string effective theory. Nucl. Phys., B440:129-170, 1995.

[60] Arthur Hebecker and Philipp Henkenjohann. Gauge and gravitational instantons: From 3-forms and fermions to Weak Gravity and flat axion potentials. arXiv:1906.07728, 2019.

[61] Anamaría Font, Alvaro Herráez, and Luis E. Ibáñez. On scale separation in type II AdS flux vacua. arXiv:1912.03317, 2019.

[62] Tom Banks. On the Limits of Effective Quantum Field Theory: Eternal Inflation, Landscapes, and Other Mythical Beasts. arXiv:1910.12817, 2019.

[63] F. Giani and M. Pernici. N=2 supergravity in ten dimensions. Phys. Rev., D30:325-333, 1984. 